Draft Version November 1, 2018

Preprint typeset using $\mathrm{I}_{\mathrm{A}}^{\mathrm{T}} \mathrm{EX}$ style emulateapj v. 10/10/03

\title{
SIMULATIONS OF MAGNETO-ACOUSTIC PULSATIONS IN ATMOSPHERES OF RAPIDLY OSCILLATING AP STARS
}

\author{
E. KhOMENKO ${ }^{1,2}$, And O. KOCHUKHOV ${ }^{3}$ \\ Draft version November 1, 2018
}

\begin{abstract}
Rapidly oscillating Ap stars exhibit an astrophysically interesting combination of strong, dipolar-like magnetic fields and high-overtone $p$-mode pulsations similar to the Sun. Recent time-resolved spectroscopy of these stars unravelled a complex picture of propagating magneto-acoustic pulsation waves, with amplitude and phase strongly changing as a function of atmospheric height. To interpret these observations and gain a new insight into the atmospheric dynamics of roAp stars we have carried out 2-D time-dependent, non-linear magneto-hydrodynamical simulations of waves for a realistic atmospheric stratification of a cool Ap star. We explore a grid of simulations in a wide parameter space, treating oscillations of the velocity, magnetic field and thermodynamic quantities in a self-consistent manner. Our simulations foster a new understanding of the influence of the atmosphere and the magnetic field on the propagation and reflection properties of magneto-acoustic waves, formation of node surfaces, and relative variation of different quantities. Our simulations reproduce all main features of the observed pulsational behavior of roAp stars. We show, for the first time, that the overall depth dependence of the pulsations in roAp atmospheres is strongly influenced by the density inversion at the photospheric base.

Subject headings: MHD - stars: magnetic fields - stars: oscillations - stars: chemically peculiar
\end{abstract}

\section{INTRODUCTION}

\subsection{General properties of roAp stars}

Rapidly oscillating Ap (roAp) stars are main sequence, late-A chemically peculiar stars showing high-overtone $p$-mode pulsations with periods between 6 and 22 minutes (Kurtz \& Martinez 2000; Kochukhov 2008). Effective temperatures of these stars span a relatively narrow range from 6400 to $8100 \mathrm{~K}$. Their atmospheres exhibit large abundance anomalies, typical of the group of $\mathrm{Sr}$ $\mathrm{CrEu}$ chemically peculiar stars. The light and iron-peak elements are normal or underabundant relative to the chemical composition of the solar photosphere, whereas concentration of the rare-earth elements (REEs) is enhanced by factors $10^{3}-10^{5}$ (Ryabchikova et al. 2004).

The presence of very strong, organized magnetic fields is another remarkable property of roAp stars. The mean field strength inferred from the polarization measurements and Zeeman splitting of spectral lines usually lies in the 1-5 kG range (Mathvs et al. 1997; Hubrig et al. 2004b; Kochukhov \& Bagnulo 2006) but can reach 25-30 kG in exceptional cases (Kurtz et al. 2006; Freyhammer et al. 2008), making Ap stars the most extreme non-degenerate magnetic stars known. These fields have simple, dipolar-like topology, often significantly inclined with respect to the stellar rotation axis (Landstreet \& Mathys 2000; Bagnulo et al. 2002). Unlike rapidly evolving, dynamo-generated magnetic fields of the Sun and late-type stars, the fields of Ap stars are most likely stable remnants of the fields acquired in the pre-main sequence evolutionary phase

\footnotetext{
Electronic address: khomenko@iac.es

${ }^{1}$ Instituto de Astrofísica de Canarias, 38205, C/ Vía Láctea, $\mathrm{s} / \mathrm{n}$, Tenerife, Spain

2 Main Astronomical Observatory, NAS, 03680, Kyiv, Ukraine

3 Department of Physics and Astronomy, Uppsala University, Box 515, SE-751 20, Sweden
}

(Moss 2004; Braithwaite \& Nordlund 2006). Ap stars also rotate significantly slower than their normal counterparts (Abt \& Morrell 1995), probably due to an enhanced braking provided by a magnetized wind and a more efficient coupling with the circumstellar disk in the pre-main sequence stage (Stẹpień 2000).

The unusual atmospheric chemistry of Ap stars is attributed to the effects of atomic diffusion (Michaud 1970). This slow process of chemical separation under the influence of competing forces of the radiative pressure and gravity is facilitated by the slow rotation and stabilizing effect of the strong magnetic field. Different diffusion velocities in the regions permeated by the horizontal and vertical magnetic field lines lead to substantial variation of chemical abundances over the surfaces of magnetic A stars (Michaud et al. 1981; Alecian \& Stift 2004). These chemical starspots are responsible for the spectacular rotational modulation of line profiles in the spectra of roAp stars, synchronized with the variation of the overall stellar brightness, photometric colors, magnetic field strength and the mean line of sight magnetic field component (Rvabchikova et al. 1997; Kochukhov et al. 2004a).

Chemical stratification in the line-forming atmospheric regions is another important consequence of the atomic diffusion in Ap stars. Detailed empirical line profile studies (Bagnulo et al. 2001; Rvabchikova et al. 2002) and self-consistent theoretical diffusion calculations (Leblanc et al. 2009) show that the light and ironpeak elements are concentrated in the lower atmosphere layers, typically below continuum optical depth at $\lambda=$ $5000 \AA \log \tau_{5}=-1: 0$. On the other hand, REEs, such as Pr and Nd which show many strong lines in the roAp spectra, are pushed by the radiative pressure to the optically thin layers above $\log \tau_{5}=-3:-4$ (Mashonkina et al. 2005, 2009).

According to the current consensus, roAp pulsations 
are driven by the $\kappa$ mechanism operating in the $\mathrm{H} \mathrm{I}$ partial ionization zone with the additional influence of chemical gradients and magnetic quenching of convection (Balmforth et al. 2001; Théado et al. 2009). The efficiency of this excitation mechanism sensitively depends on the relationship between the magnetic field, convection, and atomic diffusion. The physics of these processes is not entirely understood. Consequently, despite giving robust and informative pulsation frequency predictions (e.g., Cunha et al. 2003; Gruberbauer et al. 2008), the current roAp excitation theories are unable to account for the H-R diagram distribution of 40 known roAp stars and cannot explain their difference from apparently constant but otherwise very similar non-pulsating Ap stars.

Shortly after discovery of the $p$-mode pulsations in Ap stars a close connection between oscillations and magnetic field became obvious. Pulsational amplitude and phase were observed to follow a rotational modulation curve correlated with the field strength variation. The times of the magnetic and pulsation maxima generally coincide, while pulsation phase shows a characteristic $\pi$ radian jump when the stellar magnetic equator is closest to the line of sight (e.g., Kurtz et al. 1994). This behavior has inspired the oblique pulsator model (Kurtz 1982), which attributes roAp pulsation to a non-radial, axisymmetric, low angular degree modes aligned with the axis of dipolar magnetic field. Theoretical calculations (Saio 2005) and indirect imaging of the horizontal pulsation velocity field structure (Kochukhov 2004) have vindicated the oblique pulsator model but also revealed that the modes are substantially distorted by the magnetic field and cannot be described by a single spherical harmonic function.

\subsection{Spectroscopic pulsation signatures of roAp stars}

The advent of efficient, high-resolution spectrographs at large optical telescopes allowed extending observational analysis of roAp pulsations from traditional highspeed, broad-band photometry (see a thorough review by Kurtz \& Martinez 2000) to radial velocity (RV) measurements and studies of the individual line profile variability (see reviews by Kochukhov 2007, 2008; Kurtz 2008). These investigations revealed a number of unusual atmospheric pulsation phenomena, not present in any other type of pulsating star and not anticipated theoretically.

The most prominent and unique characteristic of the rapid spectroscopic variability of roAp stars is a large diversity of the pulsation amplitudes and phases of different spectral lines (Kanaan \& Hatzes 1998; Kochukhov \& Rvabchikova 2001; Mkrtichian et al.|2003; Elkin et al. 2005; Kochukhov 2006; Ryabchikova et al. 2007a). The narrow cores of the hydrogen Balmer lines and absorption features of the rare-earth ions usually exhibit RV amplitudes from a few hundred $\mathrm{m} \mathrm{s}^{-1}$ to several $\mathrm{km} \mathrm{s}^{-1}$. On the other hand, lines of $\mathrm{Ca}, \mathrm{Si}$ and ironpeak elements vary with amplitudes below $\sim 50 \mathrm{~m} \mathrm{~s}^{-1}$. This amplitude discrepancy is frequently accompanied by a substantial difference in the pulsation phases. In extreme cases the phase lags between RV curves of different lines reach $\pi-2 \pi$ radians (Mkrtichian et al. 2003; Rvabchikova et al. 2007a; Sachkov et al. 2008).

Equally puzzling is the pulsational bisector variation in many roAp stars. Time-series measurements performed at different intensity levels in the profiles of strong REE lines and $\mathrm{H} \alpha$ core yield systematically different results (Sachkov et al. 2004; Kurtz et al. 2005; Ryabchikova et al. 2007a,b), typically showing a large increase of the pulsation amplitude towards the line wings and occasional change of the pulsation phase across line profiles.

These spectroscopic pulsation signatures of roAp stars are difficult to reconcile with the oscillation picture expected for a normal, chemically-homogeneous stellar atmosphere. Ryabchikova et al. (2002) suggested that peculiar pulsation properties of roAp stars are related to the presence of strong atmospheric chemical composition gradients, for which there is unequivocal evidence from observations and theory alike. Chemical stratification is particularly extreme for the rare-earth ions, which levitate so high in the atmosphere that their formation heights exceed those of the hydrogen line cores. Pulsation waves propagate outwards in this chemically segregated atmosphere, gradually increasing in amplitude and consecutively perturbing the layers probed by the Fe-peak element lines, $\mathrm{H} \alpha$ and REE lines. Thus, timeresolved spectroscopic observations of roAp stars provide a vertical cross-section of $p$-modes and allow detailed study of the propagation and transformation of magnetoacoustic waves over a large range of geometrical heights.

Several time-resolved spectroscopic studies suggested that the phase-amplitude behavior of roAp pulsations show characteristics of standing waves (nearly constant phase) in deeper layers and running waves (changing phase) higher in the upper atmosphere (e.g., Kurtz et al. 2003). A few roAp stars exhibit node-like surfaces where the pulsation amplitude drops below the detection threshold and the pulsation phase undergoes a $\pi$ radian jump (Ryabchikova et al. 2007a; Sachkov et al. 2008).

High-quality spectroscopic time-series obtained for the strongest REE lines (Kochukhov \& Ryabchikova 2001) demonstrated that roAp pulsational line profile variation is also highly unusual. Weak lines of the singly ionized $\mathrm{Nd}$ and $\mathrm{Pr}$, which form in the lower part of the REE-enriched cloud, exhibit modulation expected for a low angular degree, non-radial pulsation. However, the strongest REE lines originating in the uppermost layers show asymmetric blue-to-red running waves, which are incompatible with the line profile variability pattern produced by any non-radial, horizontal pulsational velocity fluctuation. Kochukhov et al. (2007) successfully modeled this phenomenon by a combination of the RV and line width variation. Shibahashi et al. (2008) suggested an alternative explanation involving very high amplitude shock waves, unresolved by observations.

The question of the magnetic field variation with pulsation phase has been a topic of intense recent debate. Hubrig et al. (2004a) predicted magnetic variability of the order of $\delta B / B \approx 0.1$ but could not reliably detect pulsational field changes in any of the six roAp stars they surveyed with low-resolution spectropolarimetry. Leone \& Kurtz (2003) claimed a detection of $\delta B / B=0.2$ pulsational field modulation in the REE lines of the roAp star $\gamma$ Equ. However, these results were not confirmed by Kochukhov et al. (2004d) who established an upper limit of $\delta B / B=0.02$ for the same star based on a more extensive observational material. Subsequent searches of the pulsational variation of magnetic field by Kochukhov et al. (2004b); Savanov et al. 
(2006); Kochukhov \& Wade (2007) also yielded null results.

\subsection{Overview of $M H D$ wave calculations for the Sun and roAp stars}

To understand the complex physics of waves in magneto-atmospheres, numerical modeling of wave interaction with magnetic field has become a preferred approach in recent years, with the development of large computational facilities. It has an advantage over the analytical models, allowing to include magnetic field configurations of nearly arbitrary complexity and to study compound effects of several different physical agents, such as radiative losses or non-linear effects. For the Sun, where localized magnetic structures (sunspots, flux tubes) can be nearly spatially resolved in observations and studied in detail, the numerical modeling of waves in the photosphere and chromosphere of these structures has been performed for several decades, starting from e.g. Shibata (1983) and exponentially increasing in time during the last few years (Cally \& Bogdan 1997; Rosenthal et al. 2002; Bogdan et al. 2003; Hasan \& Ulmschneider 2004; De Pontieu et al. 2004; Hasan et al. 2005; Khomenko \& Collados 2006; De Pontieu et al. 2007; Steiner et al. 2007; Khomenko et al. 2008b; Hanasoge 2008; Cameron et al. 2008; Khomenko et al. 2008a; Hasan \& van Ballegooijen 2008; Moradi et al. 2009; Khomenko et al. 2009; Parchevsky \& Kosovichev 2009, without touching the literature on waves in the corona where the physical conditions are significantly different from those in the lower atmosphere). Important physical conclusions have been reached by these models. It has been demonstrated that the behavior of waves depends crucially on whether they propagate in the magnetically dominated region or in the gas pressure dominating region. The separation between these two regions is defined by the value of the plasma parameter $\beta=P_{g} / P_{m}$, the ratio between the gas pressure and the magnetic pressure. In the particular case of waves, a more adequate parameter is the ratio between the squared characteristic wave speeds, the sound speed and the Alfvén speed, $c_{S}^{2} / v_{A}^{2}$. These two parameters are related as $\left(c_{S}^{2} / v_{A}^{2}\right) / \beta=\gamma / 2=5 / 6$ (for an ideal monoatomic gas). In the magnetized stellar atmosphere the ratio $c_{S}^{2} / v_{A}^{2}$ decreases exponentially with height as the density decreases. In the solar case a typical situation is to have $c_{S}$ of the same order of $v_{A}$ at the spectral line formation heights in the photosphere or low chromosphere, depending on the field strength of the magnetic structure.

In a two-dimensional situation, when only fast and slow MHD modes exist, the slow mode is mainly magnetic in the region where $c_{S}>v_{A}$ and is mainly acoustic in the case $c_{S}<v_{A}$. The fast mode nature is the opposite. A detailed description of the fast and slow modes behavior, refraction and mode conversion in the solar atmosphere can be found in e.g. Bogdan et al. (2003); Khomenko \& Collados (2006). The slow mode is mostly field-aligned in the sense that its group velocity is parallel to the local magnetic field direction. The fast mode can propagate across the field. To establish the wave type in observations it is important to verify the height of the $c_{S}=v_{A}$ level with respect to the line formation level. Recently a lot of attention has been drawn to the phenomenon of mode transformation (Zhugzhda \& Dzhalilov 1982, 1984; Cally 2006; Cally \& Goossens 2008) taking place around the $c_{S}=v_{A}$ level. Applied to the Sun, mode transformation is believed to be responsible for the wave power reduction observed in sunspots (Cally \& Bogdan 1997) and, possibly, for the wave power redistribution over active regions (Hanasoge 2008; Khomenko 2008). Depending on the inclination of the field with respect to the wave propagation direction, the transformation can be complete or not. But in any case, new modes appear after the transformation and the wave behavior around the $c_{S}=v_{A}$ level can be extremely complex since different modes, propagating up and down, can interfere. In a threedimensional situation, the wave behavior is even more mixed, and our overall understanding is far from being complete (however, see Cally \& Goossens 2008).

Regarding the magnetized atmospheres of the roAp stars, it has been generally accepted that the magnetic field makes important effects on oscillation properties (see the recent reviews by Cunha 2007; Kurtz 2008). A number of theoretical studies have been carried out to advance understanding of the influence of magnetic field on oscillation eigenfrequencies, eigenfunctions, and on the structure of the multiplets and frequency spacings (Dziembowski \& Goode 1996; Bigot et al. 2000; Cunha \& Gough 2000; Saio \& Gautschy 2004; Saio 2005; Cunha 2006). These theoretical models were able to provide a qualitative explanation of the behavior of the frequency spacings due to the action of the magnetic field (Cunha \& Gough 2000; Saio \& Gautschv 2004). Continuing the work of Cunha \& Gough (2000), Cunha (2006) used a variational approach to calculate the magnetic perturbations to eigenfrequencies. She assumed that magnetic perturbations are introduced only in a thin layer around $c_{S}=v_{A}$ (which was called "magnetic boundary layer") changing the wave vector and introducing additional phase shifts. Note that a similar picture was used to explain the behavior of the helioseismic waves in solar spots, calling the magnetic effects "surface effects" (Braun 1997; Lindsey \& Braun 2005a,b; Moradi \& Cally 2008; Moradi et al. 2009; Khomenko et al. 2009). Saio \& Gautschy (2004) and Saio (2005) addressed the same problem with a different approach, by using expansions of the eigenfunctions into spherical harmonics (see also Dziembowski \& Goode 1996). In both approaches, it was found that, depending on its strength, the magnetic field causes cyclic effects on the pulsation frequencies. Due to the mode conversion at the magnetic boundary layer a part of the wave energy escapes to the high-frequency running acoustic waves (slow low- $\beta$ magneto-acoustic modes) or to the slow high- $\beta$ magneto-acoustic mode waves in the interior, producing mode energy losses that can become important at some particular frequencies.

As was mentioned above, the amplitudes and phases of the observed waves depend on the atmospheric height in a complex way. This behavior was largely not understood until the recent studies by Cunha (2006) and Sousa \& Cunha (2008). Unlike the Sun, oscillations observed in roAp stars come from a magnetically dominated region in the most part of the stellar atmosphere. Only in the deepest observed layers the gas pressure and 
Lorentz forces can be of the same order of magnitude for the lowest field strengths encountered in roAp stars (Cunha 2007). Due to the direct influence of the Lorentz force, running acoustic waves in the atmosphere are supposed to become field-aligned and their acoustic cut-off frequency changes as a function of the magnetic field inclination as $\omega_{c} \cos \theta$, where $\omega_{c}$ is a cut-off frequency in the absence of magnetic field (Dziembowski \& Goode 1996; Cunha \& Gough 2000). Sousa \& Cunha (2008) considered an analytical quasi one-dimensional model of the radial high-frequency pulsations (above the cut-off frequency) in a polytropic interior model of roAp stars matched to an isothermal atmosphere. They found that in the interior running magnetic waves with progressively decreasing wavelength exist (slow high- $\beta$ magnetoacoustic modes) together with nearly standing acoustic waves with much larger wavelength (fast high- $\beta$ magneto-acoustic modes). In the atmosphere the situation is the opposite: running acoustic waves with the displacement parallel to the magnetic field coexist with the nearly standing magnetic waves with displacement perpendicular to the magnetic field (slow and fast low- $\beta$ magneto-acoustic modes, respectively). A superposition of the line of sight projections of both wave components non-trivially depends on the magnetic field parameters and can possibly produce a complex spectroscopic pulsation behavior, in qualitative agreement with observations (Sousa \& Cunha 2008). For oscillations with frequencies below the cut-off frequency, the losses to the running acoustic waves in the atmosphere are restricted to latitudes where the inclination of the dipolar magnetic field is sufficient to lower down the cutoff according to $\omega_{c} \cos \theta$. For high-frequency oscillations, only a part of the energy is removed due to the running acoustic waves in the atmosphere and they can become trapped, unlike the non-magnetic case, where highfrequency waves are always propagating. The losses to the high-frequency running acoustic waves in the atmosphere were found to be the largest near the poles of the magnetic dipole where the field is nearly vertical. The losses to the running magnetic waves in the interior are the largest for fields inclined around 30 degrees. These results are similar to those from local helioseismology mode conversion studies in inclined magnetic fields by Cally (2006) and Schunker \& Cally (2006). Thus, except for regions near the poles, the high-frequency wave energy is nearly trapped. Extrapolating the results of Sousa \& Cunha (2008), waves with frequencies below the cut-off frequency are expected to be trapped at and near the poles but able to convert into running acoustic slow-mode waves with increasing inclination, so that the mode energy losses would be maximum in an annulus close to the poles (but not exactly at the poles). In all the cases, trapped waves concentrate near the magnetic equator. Note, that earlier works tried to explain the high-frequency wave reflections by an ad hoc chromospheric temperature rise (Gautschy et al. 1998), which contradicted observations since no evidence of chromospheres was found for the cool Ap stars.

The observational evidence for the lack of magnetic field variation over pulsation cycle seems to be confirmed theoretically by Saio (2005) who found that the magnetic field pulsations do not exceed the limit of $\delta B / B=$ $10^{-5}$. However, these theoretical calculations relied on an approximate analytical description of the atmospheric structure and did not extend to the low optical depths where strongly pulsating lines are formed.

\subsection{Goals of our study}

Despite this recent progress, more theoretical work is needed to reach the complete understanding of the roAp star's pulsation properties and, in particular, to explain the unusual spectroscopic variability of these stars. In this paper we use two-dimensional numerical simulations to study the properties of magneto-acoustic waves in the atmospheres of roAp stars. We take advantage of the numerical code developed by Khomenko \& Collados (2006) to model the wave propagation in magneto-atmospheres. This code was previously applied to a variety of problems of solar physics, such as the wave energy transport to the chromosphere in small-scale magnetic flux tubes, or helioseismic wave propagation though the magnetic structure of sunspots (Khomenko et al. 2008a, 2009). Our strategy is similar to the work of Sousa \& Cunha (2008). However, since the equations are solved fully numerically, we were able to relax several approximations made by these authors. We assume the waves to be excited by a radial pulsation mode below the visible surface in a stratified non-isothermal atmospheric model permeated by a constant inclined magnetic field. The code solves nonlinear equations, allowing for development of shocks in our simulations and treating the propagation of magnetoacoustic waves in a realistic cool Ap-star atmosphere. This approach allows us to relate interesting pulsation properties with the formation heights of important diagnostic lines, thereby bridging the gap between the complex observational picture of roAp pulsation and theoretical models, previously limited to simplistic analytical atmospheric structures.

Our goal is to create a more generalized picture and to study in detail the effects of the magnetic field strength and inclination, and the temperature stratification on the observed pulsation amplitudes and phases. We analyze the behavior of individual magneto-acoustic modes at different latitude locations and at different frequencies, both below and above the atmospheric cut-off frequency. The signatures of these waves in the discintegrated signal are also studied. Our analysis is not limited just to the velocity oscillations, but we also study self-consistently the temporal behavior of thermodynamic parameters and the of magnetic field vector.

Our simulations are suitable for detailed spectral synthesis and a direct comparison to observations. However, we defer this work to subsequent papers. Below we concentrate on the analysis of the physical properties of magneto-acoustic waves under different conditions and try to address several key questions: why such a diverse pulsation wave behavior is observed in the atmospheres of roAp stars, depending on the spectral line (i.e. the height in the atmosphere); which physical mechanisms determine the presence of the node surfaces, rapid growth of the amplitude and change of the pulsation phase with height, mixture of an upward and a downward wave propagation; what kind of temperature, density and magnetic field variations accompanies RV oscillations, etc.

\section{LOCAL MHD SIMULATIONS}



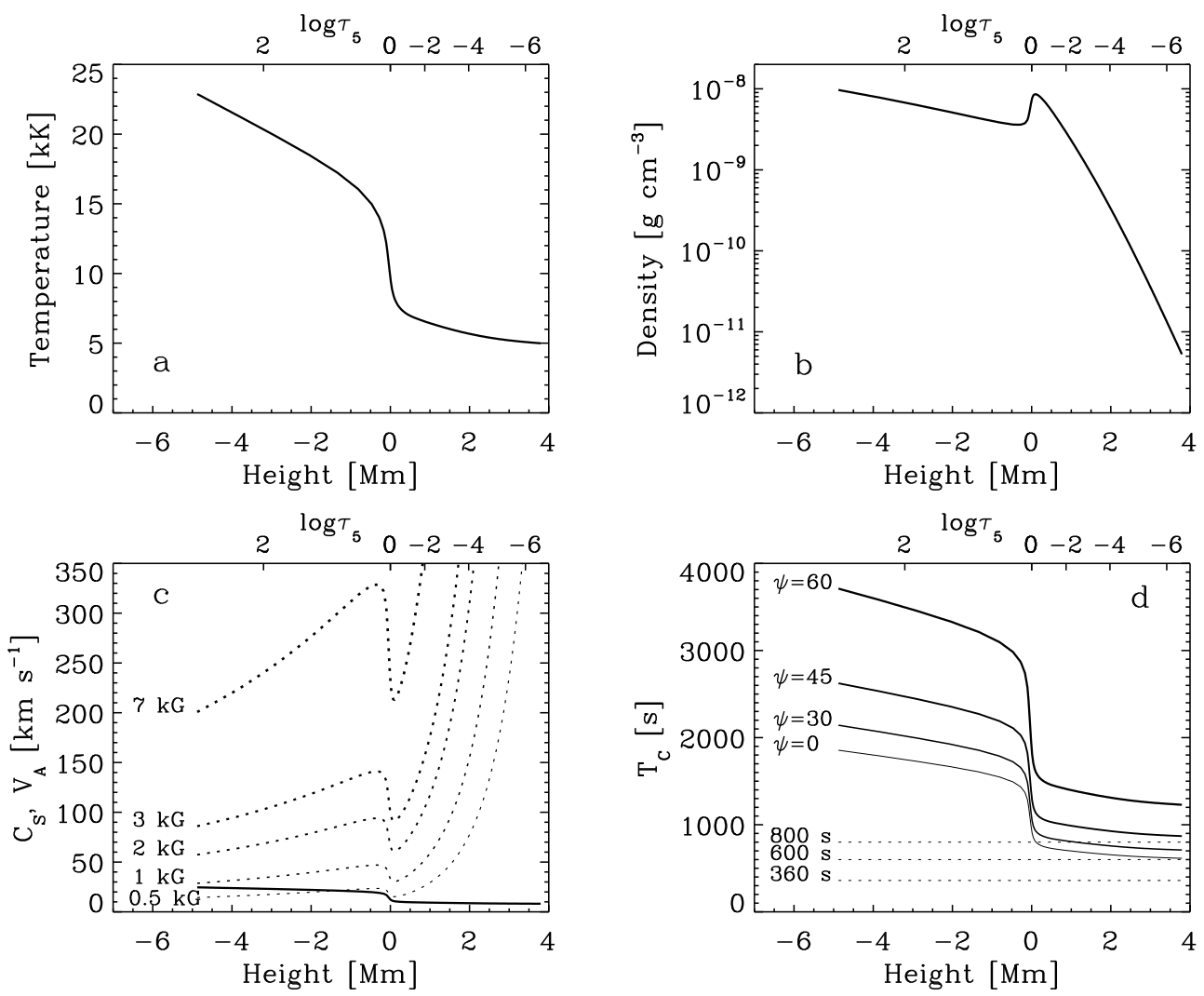

Fig. 1.- Equilibrium model atmosphere. (a) Temperature. (b) Density. (c) Solid line: acoustic speed; dotted lines: Alfvén speed for the values of the magnetic field marked with numbers. (d) Solid lines: cut-off period $T_{c}=2 \pi /\left(\omega_{c} \cos \psi\right)$, where $\psi$ is the magnetic field inclination and $\omega_{c}=\gamma g / 2 c_{S}$. Dotted lines: periods of the driver. In all panels zero height corresponds to $\log \tau_{5}=0$. The optical depth scale is given by the upper axis.

The global magnetic field of roAp stars can be approximated to a first order as a magnetic dipole (Bagnulo et al. 1995; Kochukhov et al. 2004a). The characteristic wavelength of oscillations can be estimated as $\lambda \sim c_{S} T$, where $T$ is the pulsation period. For a typical model atmosphere of roAp star $c_{S}$ is approximately a few tens of $\mathrm{km} \mathrm{s}^{-1}$, depending on the atmospheric height, and $T$ is of the order of ten minutes, making the wavelength to be of the order of $10^{1} \mathrm{Mm}$. This number is negligible compared to the stellar radius and to the characteristic scale of the variations of the magnetic field. Based on these considerations we take a local approach in the simulations, assuming that the magnetic field is locally homogeneous and inclined.

Given that the observed pulsations are of low degree $\ell$, we assume that at the base of our simulation domain (below the visible surface) the oscillations are excited by a plane-parallel perturbation with mostly vertical displacement. For the dipolar field and modes with zero azimuthal order $(\mathrm{m}=0)$ the pulsations are axisymmetric. The simulated waves propagate in a plane defined by the magnetic field vector and the gravity vector. For the dipolar field the pulsations are axisymmetric and their properties depend on the magnetic latitude, but not on the azimuthal angle. Apart from this, the properties of oscillations in the horizontal direction are not constrained by any assumption.

Dziembowski \& Goode (1996); Cunha \& Gough (2000); Cunha (2006); Sousa \& Cunha (2008) also solved a local problem in a plane-parallel atmosphere with a locally uniform magnetic field, allowing oscillations to have the displacement vector in the two-dimensional plane. The difference between our approach and the approach of these authors is that our analysis is not limited to low-amplitude oscillations and allows arbitrary variations of the velocity in horizontal direction in contrast to quasi-radial pulsations considered in the aforementioned papers. We focus entirely on the propagation of waves in the stellar atmosphere and a small portion of sub-surface layers and do not consider excitation of the pulsations and mode transformation in the stellar interior.

\subsection{Numerical method}

We use the numerical MHD simulation code that was developed during the last years at the Instituto de Astrofísica de Canarias (IAC) to study the wave propagation in different magnetic structures. The description of the code can be found in Khomenko \& Collados (2006); Khomenko et al. (2008b). The code solves the basic nonlinear equations of the ideal MHD, written in conservative form:

$$
\frac{\partial \rho}{\partial t}+\vec{\nabla} \cdot(\rho \vec{V})=0
$$

$$
\frac{\partial(\rho \vec{V})}{\partial t}+\vec{\nabla} \cdot\left[\rho \vec{V} \vec{V}+\left(P+\frac{\vec{B}^{2}}{8 \pi}\right) \mathbf{I}-\frac{\vec{B} \vec{B}}{4 \pi}\right]=\rho \vec{g},
$$




$$
\begin{gathered}
\frac{\partial E}{\partial t}+\vec{\nabla} \cdot\left[\left(E+P+\frac{\vec{B}^{2}}{8 \pi}\right) \vec{V}-\vec{B}\left(\frac{\vec{B} \cdot \vec{V}}{4 \pi}\right)\right]=\rho \vec{V} \cdot \vec{g}+\rho Q, \\
\frac{\partial \vec{B}}{\partial t}=\vec{\nabla} \times(\vec{V} \times \vec{B}),
\end{gathered}
$$

where $\mathbf{I}$ is the identity tensor and $E$ is the total energy:

$$
E=\frac{1}{2} \rho V^{2}+\frac{P}{\gamma-1}+\frac{B^{2}}{8 \pi} .
$$

All other symbols have their usual meaning. In these equations the gravity $\vec{g}$ is taken constant with a value corresponding to that at the stellar surface. In the present version of the code, the radiative energy losses, $Q$, can be included according to the Newton cooling law. However, we postpone the study of non-adiabatic effects to the future.

The code solves the non-linear MHD equations for perturbations, that are obtained by subtracting the equations of initial magnetostatic equilibrium from Eqs. 114, Note that this procedure is not equivalent to a usual linearization of the equations since we maintain all the non-linear terms in the equations for perturbations. In the simulations described above we use the equation of state of an ideal gas.

The boundary condition issue is very important for wave simulations since artificial reflections at the boundaries can distort the physical results and limit the duration of the simulations. Our code makes use of a Perfectly Matching Layer (PML) boundary condition (Berenger 1994) that efficiently absorbs the incoming waves at the boundaries and prevent their return into the simulation domain, using relatively few grid points. In the simulations described below, the horizontal domain boundaries are periodic and the top boundary has a PML layer. At the bottom boundary of the simulation domain we drive sine waves with a given period by means of the exact analytical solution for the radial acoustic-gravity waves in an isothermal stratified atmosphere (Mihalas \& Mihalas 1984):

$$
\begin{gathered}
V_{z}=V_{0} \exp \left(\frac{z}{2 H}\right) \sin \left(\omega t-k_{z} z\right) \\
\frac{\delta \rho}{\rho}=\frac{V_{0} k_{z}}{\omega}|R| \exp \left(\frac{z}{2 H}\right) \sin \left(\omega t-k_{z} z+\phi_{R}\right) \\
\frac{\delta P}{P}=\frac{V_{0} k_{z} \gamma}{\omega}|P| \exp \left(\frac{z}{2 H}\right) \sin \left(\omega t-k_{z} z+\phi_{P}\right)
\end{gathered}
$$

where $H=P /(d P / d z)$ is the pressure scale height and the amplitudes and relative phase shifts of the pressure and density perturbations are given by:

$$
\begin{gathered}
|R|=\sqrt{\frac{1}{\left(2 H k_{z}\right)^{2}}+1} \\
|P|=\sqrt{\frac{1}{\left(2 H k_{z}\right)^{2}}\left(\frac{\gamma-2}{\gamma}\right)^{2}+1} \\
\phi_{R}=\arctan \left(\frac{1}{2 H k_{z}}\right) \\
\phi_{P}=\arctan \left(\frac{\gamma-2}{\gamma} \frac{1}{2 H k_{z}}\right)
\end{gathered}
$$

Given the wave frequency $\omega$, the vertical wave vector $k_{z}$ is found from the usual dispersion relation for acousticgravity waves $k_{z}=\sqrt{\left(\omega^{2}-\omega_{c}^{2}\right)} / c_{S}$. The wave vector $k_{z}$ is zero if the driving frequency is below the local cut-off frequency.

As the atmosphere at the bottom boundary is not isothermal, the chosen form of driver is not an exact solution for this layer. The vertical dependence in equations (6) 8) would be slightly different including the temperature gradient. We have checked the implications of this form of driving on the solution developed in the physical domain. We found that varying artificially the vertical dependence in the equations (6 8) till the extreme case of oscillations in the non-stratified atmosphere has negligible influence on the solution in the physical domain. This gives us confidence that the numerical solution is not biased by this particular form of driving.

\subsection{Model atmosphere and simulation setup}

As unperturbed equilibrium model atmosphere we adopt a model computed with the help of the opacity-sampling model atmosphere code LLmodels (Shulyak et al. 2004). This plane-parallel, LTE model has effective temperature $T_{\text {eff }}=7750 \mathrm{~K}$ and gravitational acceleration $\log g=4.0$ (cgs units). The model takes into account abundance anomalies typical of cool magnetic Ap stars and is part of the grid presented by Kochukhov \& Shulvak (2008). The dipolar magnetic field is force-free and, therefore, does not influence the hydrostatic equilibrium of the model.

Some parameters of the model are displayed in Fig. 1. It gives the temperature, density, characteristic speeds and cut-of period stratifications as a function of geometrical height and continuum optical depth $\log \tau_{5}$. Several features of the model should be emphasized for better understanding the simulations. The temperature has a very strong gradient at the surface decreasing almost a factor of three in $0.5 \mathrm{Mm}$. This has consequences on the critical period $T_{c}$ and on the acoustic speed $c_{S}$, which both fall abruptly at the atmospheric base. The density stratification has a bump around zero kilometers $\left(\log \tau_{5}=0\right)$, which appears due to the hydrogen ionization in these layers. Due to this bump and due to the temperature gradient, we can expect strong reflection of waves coming from the interior to the surface. As a consequence of the density stratification, the Alfvén speed (Fig. 15) has a complex behavior showing a local minimum around zero kilometers.

In the present paper we perform a grid of simulations made for different combinations of three parameters: local magnetic field strength, its inclination and driving period. The magnetic field strength grid has 5 values: $B_{0}=0.5,1,2,3$ and $7 \mathrm{kG}$. The inclination grid has 4 values: $\psi=0,30,45$ and 60 degrees to the local vertical. And the period grid has 3 values: 360, 600 and $800 \mathrm{~s}$. This makes in total 60 models. In this way we span the typical range of magnetic field strengths and pulsation periods observed in roAp stars. The initial amplitude of oscillations at the base of the simulation domain was $V_{0}=100 \mathrm{~m} \mathrm{~s}^{-1}$ for all simulations. Through this paper we take positive sign for velocity corresponding to a redshift (downflow).

Note that we do not introduce any latitudinal dependence of the driving velocity. We use the same form 

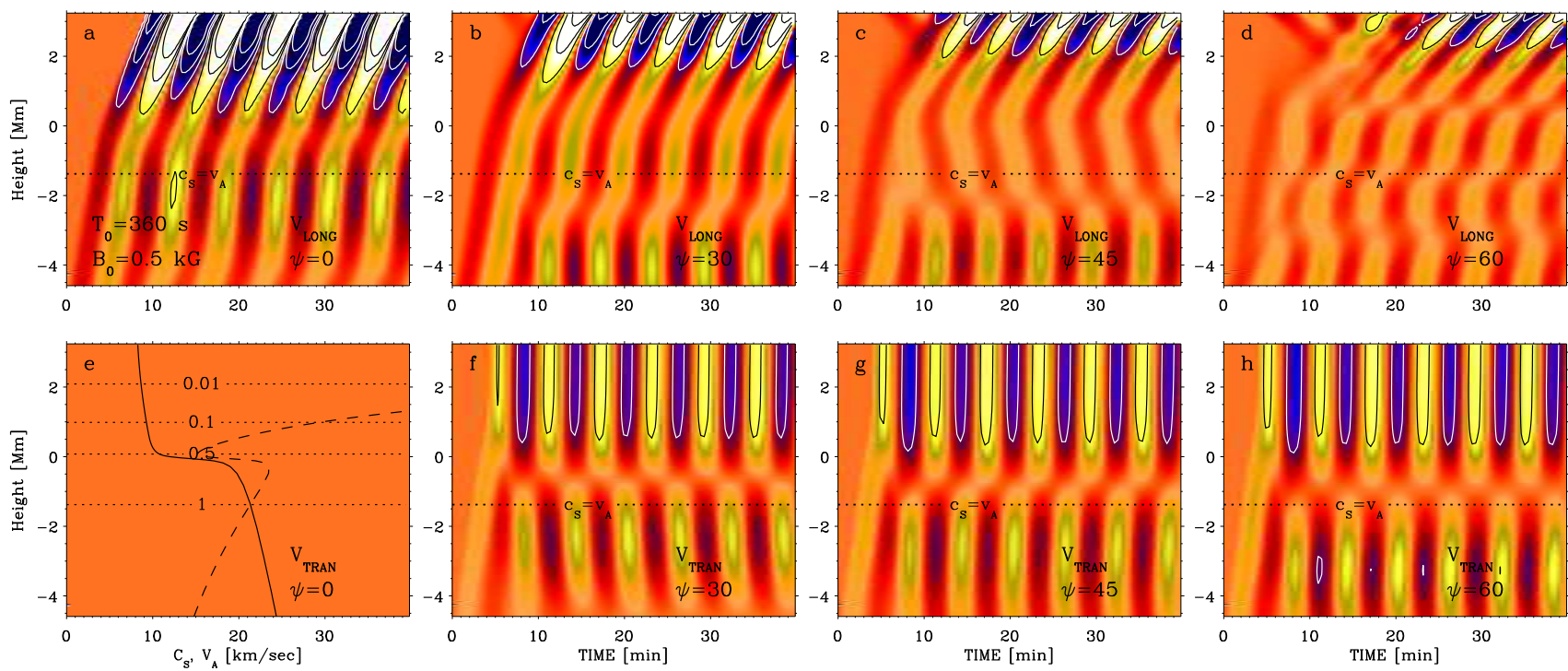

FIG. 2.- Height-time variations of the longitudinal (parallel to the field, top) and transversal (perpendicular to the field, bottom) velocities for $B_{0}=0.5 \mathrm{kG}, T_{0}=360 \mathrm{~s}$ and inclinations equal to $\psi=0,30,45$ and $60^{\circ}$. Yellow and white colors mean positive velocities and dark red and blue colors mean negative velocities. The velocity contours of $|V|=(1,0.5,0.3) \mathrm{km} \mathrm{s}^{-1}$ are overplotted as solid lines. Zero height corresponds to the photospheric base $\left(\log \tau_{5}=0\right)$. Dotted lines marked with numbers are contours of constant $c_{S}^{2} / v_{A}^{2}$. Height dependences of the sound speed $c_{S}$ (solid line) and Alfvén speed $v_{A}$ (dashed line) are plotted over the left bottom panel.
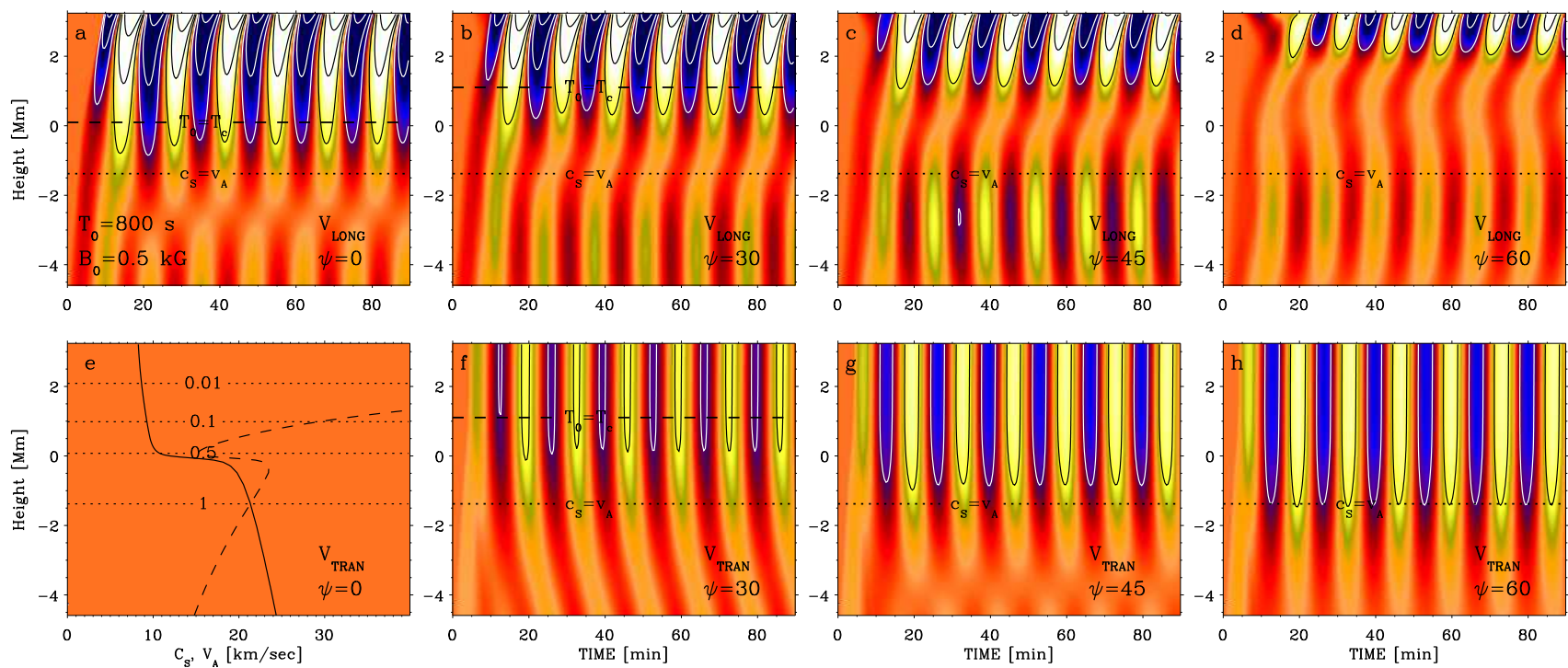

Fig. 3.- Same as Fig. 2 but for $T_{0}=800 \mathrm{~s}$. The dashed line marks the location where the wave period is equal to the cut-off period $T_{0}=T_{c} / \cos \psi$.

of the driver (Eq. 6 8) for all inclinations of the magnetic field, i.e. at all latitudes of the star. In other words, we study the response of the stellar atmosphere to an acoustic wave perturbation introduced from below. By doing so we neglect the possible presence of other wave types at heights of the lower boundary of our simulation domain. For the range of the considered magnetic field strength, the low boundary is the region where the asymptotic solutions for different decoupled modes still can not be safely applied. The weight of the different modes at these heights depends on their propagation, reflection and transformation in the deeper layers and is apriory unknown. Several theoretical studies (e.g. Sousa \& Cunha 2008) show that the presence of magnetic boundary layer has strong implications on the latitudinal dependence of the amplitudes and phases of waves at the bottom of the atmosphere. However, we can not use the theoretical eigenfunctions calculated in such studies directly in application to our simulations, as the atmospheric model considered in them is different from ours. Giving these limitations, the reader has to keep in mind that the results of our calculations, as for the relative amplitude and phase distributions of waves at different latitudes (Sect. 3.1.0), are strictly valid only assuming acoustic waves at the bottom of the atmosphere with the latitude-independent properties.

Fig. 1t gives the Alvén speed for the $B_{0}$ grid values. As we see from the figure, already for a field strength as weak as $1 \mathrm{kG}$, the Alfvén speed exceeds the acoustic speed in the whole height range, making the atmosphere magnet- 

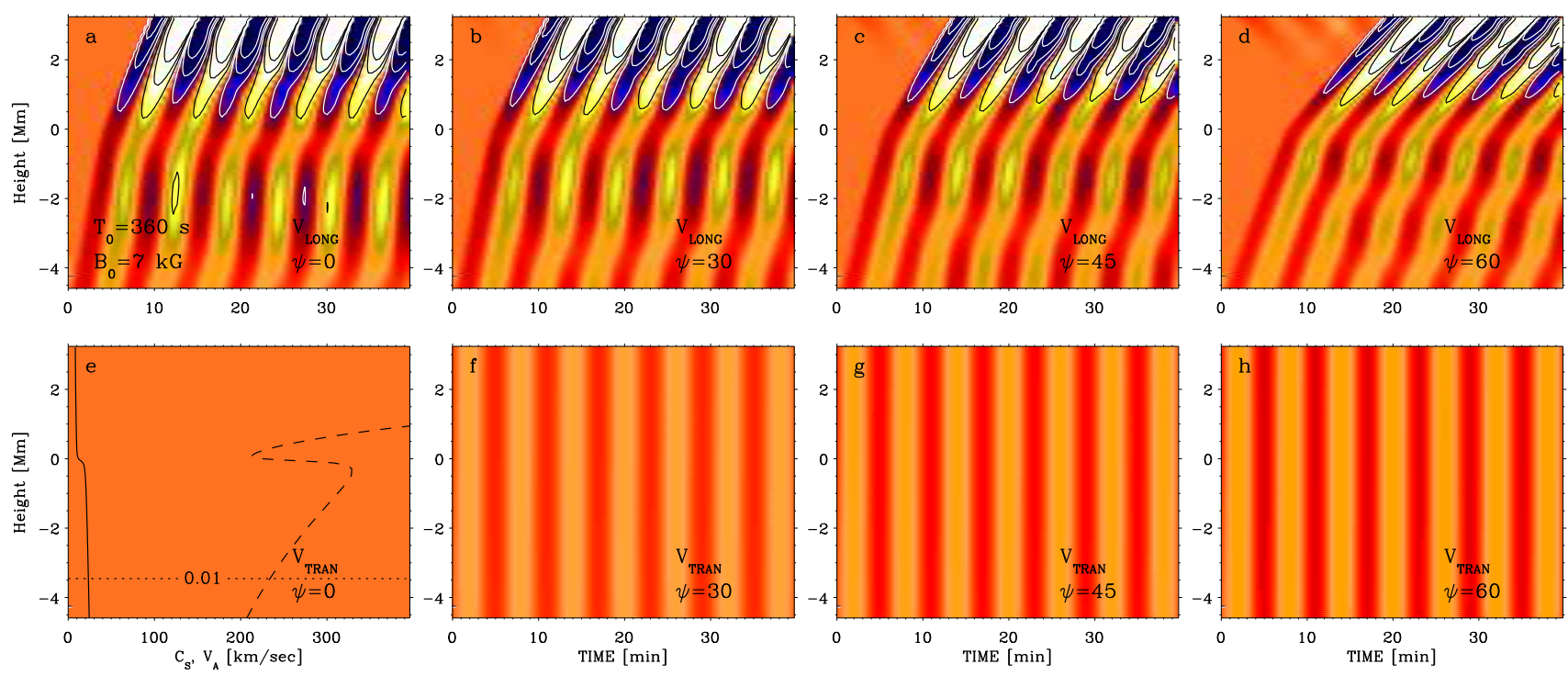

Fig. 4.- Same as Fig. 2 and Fig. 3 but for $B_{0}=7 \mathrm{kG}$ and $T_{0}=360 \mathrm{~s}$.
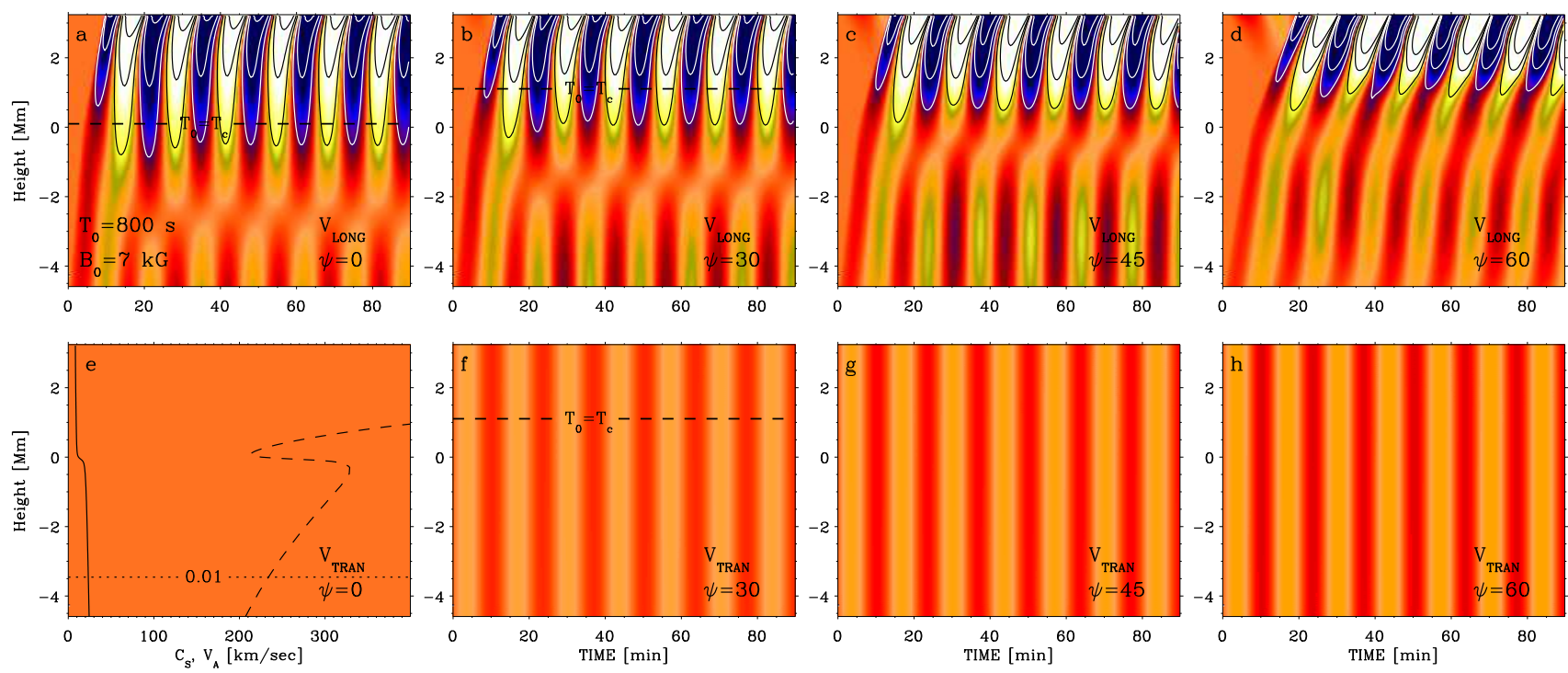

Fig. 5.- Same as Fig. 2 and Fig. 3 but for $B_{0}=7 \mathrm{kG}$ and $T_{0}=800 \mathrm{~s}$.

ically dominated. Only for $B_{0}=0.5 \mathrm{kG}$ the plasma is pressure dominated below $-1 \mathrm{Mm}$. Thus, we expect to see some mode transformation in the corresponding simulations.

In the magnetically dominated atmosphere, the cutoff frequency changes with the magnetic field inclination angle as the acoustic wave propagation becomes fieldaligned. Fig. 1 id illustrates acoustic cut-off periods for the inclination grid of our simulations. This figure also shows the driving periods $T_{0}$. In our model atmosphere waves with $T_{0}=360 \mathrm{~s}$ are propagating at all heights. However, waves with $T_{0}=800 \mathrm{~s}$ are propagating only in the interior and become evanescent at the surface. Thus, some reflection of the 800 s-period waves is expected at the surface.

Through the rest of the paper, we describe the results of our simulations in terms of fast and slow magnetoacoustic-gravity waves (note that we use a more con- cise notation, i.e. "magneto-acoustic"). These waves are the two possible solutions of the MHD equations (1) (4), one with a larger propagation speed and one with a lower propagation speed. In an academic case of a homogeneous atmosphere that can be described by single values of Alfvén speed $v_{A}$ and the sound speed $c_{S}$, the waves maintain their fast or slow properties globally in the whole domain. As we consider the case of nonhomogeneous vertically stratified atmosphere, the propagation speeds of the two solutions change with height. In the regions in the atmosphere where $v_{A} \ll c_{S}$ or $v_{A} \gg c_{S}$ the two solutions are expected to recover their asymptotic behaviour identified with classical slow and fast waves of the homogeneous atmosphere. In the intermediate situation when $v_{A}<c_{S}$, one can still identify the fast mode as mostly acoustic and a slow mode as mostly magnetic. The opposite is true in the region where $v_{A}>c_{S}$ (see, e.g. Cally 2001; Bogdan et al. 2003; 
Khomenko \& Collados 2006, where such a classification is applied). The modes can be considered as "coupled" in the region where $v_{A} \approx c_{S}$. The later term means that their properties become close. Even in this situation we preserve the notation "fast" and "slow", as the propagation speed of one solution is always larger than the other one, except when $v_{A}=c_{S}$ strictly.

\section{RESULTS}

\subsection{Velocity variations}

According to Fig. 11 the simulated part of the atmosphere is in the magnetically dominated regime down to field strength of $1 \mathrm{kG}$. In this regime the natural reference system for oscillations is the one defined by the magnetic field. Below in this section we discuss oscillations of velocity parallel to the field and perpendicular to the field ( $V_{\text {long }}$ and $V_{\text {tran }}$, respectively). This way the two, fast and slow, magneto-acoustic modes can be distinguished (Khomenko \& Collados 2006).

As mentioned above, the clear distinction between the two modes can be done only in the situation where $v_{A} \gg c_{S}$ or $v_{A} \ll c_{S}$. In the $v_{A} \gg c_{S}$ limit, the slow mode is mostly an acoustic-gravity wave guided along the magnetic field lines and is visible in the longitudinal velocity and in the gas pressure variations. The fast mode is magnetic and is visible in the transversal velocity as well as in the variations of the magnetic field vector. In the $v_{A} \ll c_{S}$ limit, the slow mode (now magnetic) is better visible in the transverse velocity variations and in the magnetic field vector variations. The slow waves in this region are quasi-Alfvénic in character (Bogdan et al. 2003), thus, the principal motion is transverse to the magnetic field and the propagation direction is along the magnetic field. While the fast mode in the $v_{A} \ll c_{S}$ limit is mostly acoustic and has no relation to the magnetic field. It can have both longitudinal and transverse velocity components accompanied by pressure fluctuations. In the intermediate situation $\left(v_{A}<c_{S}\right.$ or $v_{A}<c_{S}$ ) the modes can not be so clearly distinguished, but still preserve their mostly acoustic or mostly magnetic behaviour, depending on the region. Note that we keep the discussion in terms of $V_{\text {long }}$ and $V_{\text {tran }}$ projections even in this intermediate situation since it is convenient to keep the same notation over the whole atmosphere. It allows the analysis of the different mode behavior to be done in a clear way.

Figs. 2 5 show $V_{\text {long }}$ and $V_{\text {tran }}$ velocities as a function of time and vertical coordinate. We take for illustration four representative cases of simulations. In the first case shown in Fig. 2, the magnetic field strength is weak $\left(B_{0}=0.5 \mathrm{kG}\right)$ and the wave period $\left(T_{0}=360 \mathrm{~s}\right)$ is well below the acoustic cut-off period. In this simulation, half way though the atmosphere the high frequency waves meet the layer where $c_{S}=v_{A}$ and we expect to observe wave mode transformation and reflections. In the second case, shown in Fig. 3, the magnetic field is similarly weak, but the wave period is above the acoustic cut-off period at the upper part of the atmosphere $\left(T_{0}=800\right.$ s), giving the possibility to study the behavior of the evanescent waves. The third case (Fig. 41) illustrates the propagation of the high-frequency waves $\left(T_{0}=360 \mathrm{~s}\right)$ in a strong magnetic field $\left(B_{0}=7 \mathrm{kG}\right)$. Finally, the last case (Fig. 5) is for low-frequency evanescent waves $\left(T_{0}=800\right.$ s) in the strong magnetic field. In all the cases we show simulations at four magnetic latitude locations where the inclination of the magnetic field to the local vertical is $\psi=0$ (magnetic pole), 30,45 and $60^{\circ}$. Note that the format of these figures is such that larger inclination of the ridges means lower propagation speeds of waves and that vertical ridges mean infinite propagation speed, i.e. stationary waves.

\section{High-frequency waves in the weak field}

Fig. 2a shows that, as expected, in the vertical field $\left(\psi=0^{\circ}\right)$ only one type of wave exists with the velocity aligned to the field. The vertical driver excites vertically propagating fast magneto-acoustic mode that is essentially acoustic at the bottom of the simulation domain where $c_{S}>v_{A}$. Propagating upwards this wave encounters the layer where $c_{S}=v_{A}$ and is completely transformed into the slow magneto-acoustic wave (also acoustic). This mode only has longitudinal velocity component. It can be appreciated how the propagation speed of waves decreases above $0 \mathrm{~km}$, in agreement with the rapid decrease of the acoustic speed (Fig. 2 ). Fig. 6a and e show the amplitudes and phases of the $V_{\text {long }}$ and $V_{\text {tran }}$ velocities for the simulations with $B_{0}=0.5 \mathrm{kG}$ and $T_{0}=360 \mathrm{~s}$. The amplitude of the longitudinal velocity in the $\psi=0^{\circ}$ case (black lines) does not increase monotonically, but has two local minima and a local maximum at height around $-2 \mathrm{Mm}$. This structure is formed by partial reflection of the acoustic wave because of the strong density, temperature and, thus, sound speed, gradient at $Z=0 \mathrm{~km}$. As the frequency of the wave is above the cut-off frequency in the whole domain (see Fig. 10 this reflection can not be due to the cut-off frequency effects. An instructive discussion on the partial reflection of highfrequency (above the cut-off) waves in the atmosphere of Sun and stars is presented in Balmforth \& Gough (1990). In this paper the authors consider, among others, the case of a polytropic interior matched to an isothermal atmosphere with a discontinuous jump in the sound speed. They come to the conclusion that reflection of high-frequency waves in such a jump can be significant. Their schematic model gives a very good approximation to our model atmosphere where the jump in the sound speed is produced at the photospheric base due to the hydrogen ionization. In the solar case, partial reflections of waves from the photospheric temperature gradient were also studied by e.g. Marmolino et al. (1993). After the reflection the waves become partially trapped between -3 and $0 \mathrm{Mm}$. This is evident from their phase behavior (black line in Fig. 6e) that is almost constant at these heights. Below and above the trapped zone, the acoustic waves are propagating upwards and their phase is monotonically increasing with height.

The case of the inclined field is substantially more complex. The vertical driver in the inclined field excites a mixture of the fast and slow magneto-acoustic modes and their relative contribution depends on the field inclination. On their way up the fast and slow modes encounter first the transformation $c_{S}=v_{A}$ layer. In the case of the inclined field the fast-to-slow (and vice versa) mode transformation is not complete and depends on the wave frequency and the angle between the wave vector and the magnetic field vector. According to Cally (2006) the higher the frequency, the smaller is the cone of angles where the fast-to-slow mode transformation is effective. 
The longitudinal velocity variations show that the fast mode generated by the driver in the $c_{S}>v_{A}$ region is partially transformed into the slow mode in the $c_{S}<v_{A}$ region (Fig. 2b, c and d). The comparison of the velocity amplitudes in Fig. 6] for the case of three different inclinations $\psi$ indicates that the part of the driver energy that finally reaches the upper layer in the form of the slow mode might decrease with the inclination angle. This happens because fast-to-slow mode transformation at the $c_{S}=v_{A}$ layer becomes less and less effective with increasing magnetic field inclination, in agreement with Cally (2006). Note, that, in principle, this general trend might be modified considering more periods in the simulations. As shown by Cunha \& Gough (2000), the efficiency of the mode coupling and its relation to the inclination of the magnetic field depends on frequency in a highly non-linear manner.

The transversal velocity variations in Fig. 2f, g and h show that the slow (magnetic) mode is produced by the driver in the $c_{S}>v_{A}$ region. Preceding the results from Sect 3.2 the presence of the slow magnetic mode is not only deduced from the transversal velocity variations but also from the significant magnetic field variations present in this region (see Fig. 12 for $T_{0}=600 \mathrm{sec}$, the results are similar for $\left.T_{0}=360 \mathrm{sec}\right)$. The slow magnetic mode is partially transformed into the fast magnetic mode in the $c_{S}<v_{A}$ atmosphere. Due to the drastic increase of the Alfvén velocity in the upper layers the propagation speed of the fast mode becomes very large. The fast mode is partially reflected from such strong Alfvén speed gradient region and passes again through the transformation region. This generates additional fast and slow modes that interfere with the upcoming waves. The downward slow mode propagation below $-0.5 \mathrm{Mm}$ is clearly seen in Fig. 2f and Fig. 6e (red dashed line). The node surface is formed due to the interference of the upwardly propagating slow waves and downwardly propagating slow waves slightly above the $c_{S}=v_{A}$ height. The exact position of this surface depends on the vertical wavelength of the waves. Depending on the field inclination, the mixture of the modes generated by the driver and propagating upwards, those produced after the multiple mode transformations and those partially reflected due to the strong temperature gradient creates complex interference pictures, particularly evident in the case of the largest field inclination $\psi=60^{\circ}$ (Fig. 2 d). The number and location of the node layers depends on the field inclination, both for $V_{\text {long }}$ and $V_{\text {tran }}$, and is particularly complex in the case of weak field and low period (and, thus, low wavelength) waves (Fig. 6).

\section{Low-frequency waves in the weak field}

The case of the low-frequency waves propagation in the weak field is illustrated in Fig. 3 and Fig. 7 (panels a and e). In this case, apart from the transformation layer, we have a layer where the wave frequency becomes larger that the cut-off atmospheric frequency. For waves with $T_{0}=800 \mathrm{~s}$, this layer is located in the atmosphere where $c_{S}<v_{A}$ and, thus, its location depends on the field inclination.

In the vertical field $\left(\psi=0^{\circ}\right.$, Fig. 33 $)$ the propagation properties are similar to the case described above. The waves with $800 \mathrm{~s}$ period are reflected around $Z=0 \mathrm{Mm}$ due to the cut-off frequency effects. Due to this reflec- tion, a node structure appears at about $-2.5 \mathrm{Mm}$ and a standing wave pattern is formed by the interference of the upcoming and downward reflected waves. Above $Z \approx 0 \mathrm{Mm}$, the $800 \mathrm{~s}$ period waves are evanescent. The evanescent wave pattern is evident from the phase and amplitude behavior of waves in Fig. $7 \mathrm{a}$ and e. The phase is almost constant with height, except for the $\pi$ radian jump at $Z \approx-2.5 \mathrm{Mm}$, and the amplitude increase is much less that in the case of $360 \mathrm{~s}$ period waves. One may note a propagating behavior of the waves above 2 $2.5 \mathrm{Mm}$.

In the inclined field, similar to the previous case, a mixture of the fast and slow magneto-acoustic modes is generated at the lower boundary of the simulation domain, propagating upwards. After the transformation layer, these modes are partially transformed and partially transmitted. The slow magneto-acoustic modes in the $c_{S}<v_{A}$ atmosphere are now affected by the cut-off frequency effects. For the field inclination of $\psi=30^{\circ}$ the position of the cut-off height has been shifted to $Z \approx 1$ $\mathrm{Mm}$ and the waves become evanescent above this height, unlike the case of $T_{0}=360 \mathrm{~s}$. With increasing inclination, the cut-off height is moved higher up in the atmosphere outside the simulation domain and the waves become propagating upwards. This behavior is clear from the phase variations above $Z=1 \mathrm{Mm}$ (Fig. 77). The change of the slope of the ridges in Fig. $3 \mathrm{~b}, \mathrm{c}$ and d with increasing inclination is due to the projection effects of the field-aligned propagation of slow mode waves.

Fig. 3f, g and h show how the slow magneto-acoustic waves ${ }^{4}$ generated by the driver at the low boundary in the $c_{S}>v_{A}$ region are partially transformed to the fast magneto-acoustic waves in the $c_{S}<v_{A}$ atmosphere and again adopt a quasi-evanescent behavior due to the rapid increase of the Alfvén speed. After the partial reflection of the fast magneto-acoustic waves, downward propagating waves are clearly seen below $c_{A}=v_{A}$ height. Similar to the $T_{0}=360 \mathrm{~s}$ case, these downward propagating waves are slow modes generated after the secondary transformation at the $c_{A}=v_{A}$ layer. The phase and amplitude behavior is qualitatively similar for the $T_{0}=360$ and $800 \mathrm{~s}$ waves (compare dashed curves in Fig.6a, e and Fig. $7 \mathrm{a}, \mathrm{e}$ ). The different pattern and node locations are due to the different vertical wavelengths of these waves. The height pattern is much smooth and continuous for the waves with larger periods.

\section{High-frequency waves in the strong field}

The results of the simulations of the high-frequency wave propagation in the strong magnetic field are illustrated in Fig. 4 and Fig. 6 (panels d and h). The case of the strong field is much easier to analyze since the wave propagation always occur in the magnetically dominated regime and there is no mode transformation. In the high-frequency case there are also no cut-off frequency effects and the slow (acoustic) waves are propagating at all heights.

In the vertical field the driver generates slow magnetoacoustic waves. In the magnetically dominated atmosphere these waves are essentially acoustic and their

\footnotetext{
4 Again, the slow mode in this region can be considered as mainly magnetic as the variations in the transversal velocity are accompanied by significant magnetic field variations.
} 

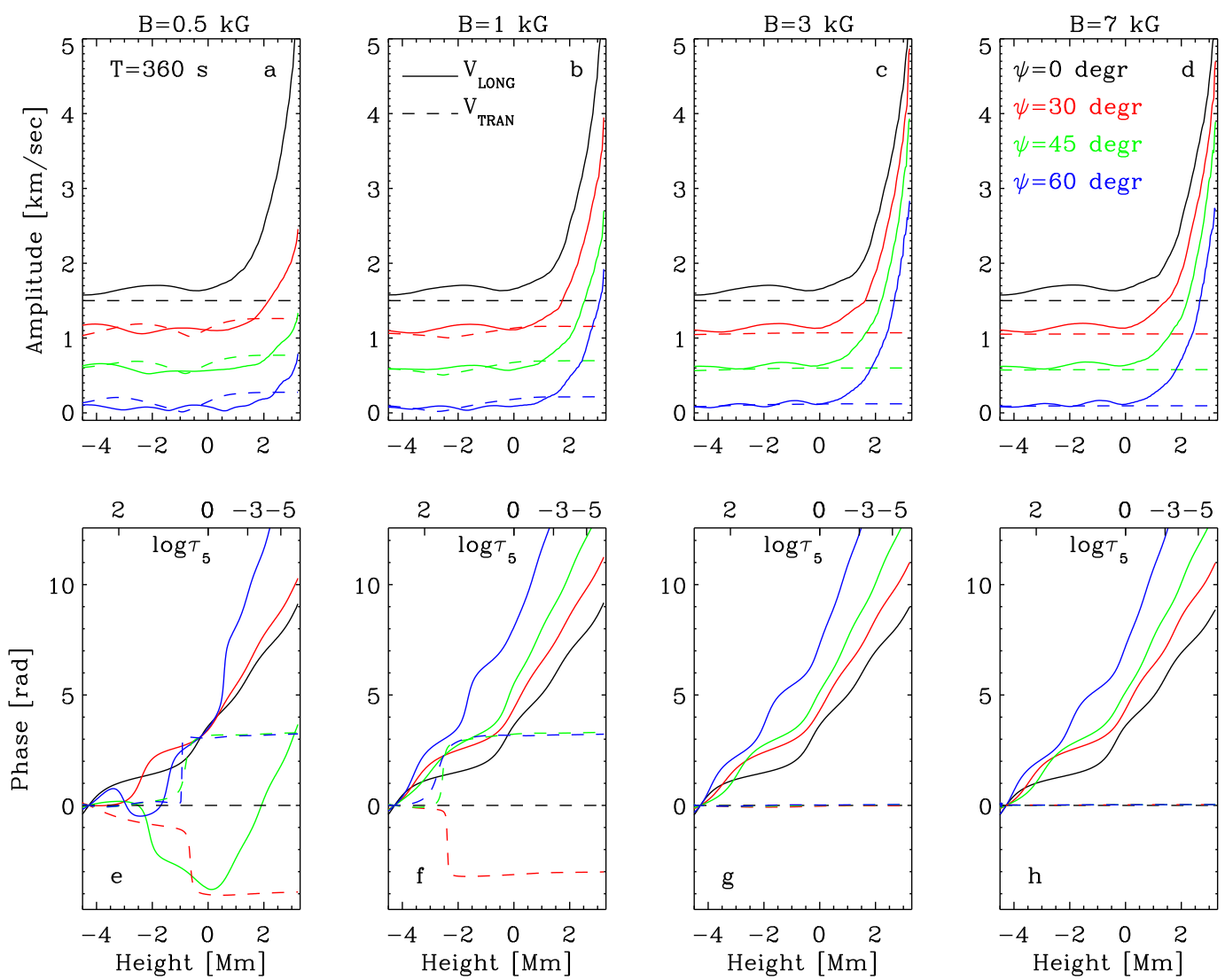

FIG. 6.- Amplitudes (top) and phases (bottom) of the longitudinal (solid lines) and transversal (dashed lines) velocities as a function of height for simulations with $B_{0}=0.5,1,3$ and $7 \mathrm{kG}$ and at different inclinations $\psi$ (indicated in the figure). The results are for the oscillation period $T_{0}=360 \mathrm{~s}$. The amplitude curves for each $\psi$ are separated by $0.5 \mathrm{~km} / \mathrm{sec}$ for better visualization. The function fitted to the simulations is $A \sin (\omega t+\varphi)$, so that larger phase $\varphi$ indicates a later time of pulsation maximum.

propagation properties are identical to the case of $B_{0}=$ $0.5 \mathrm{kG}$, as the vertical magnetic field does not affect to the first order the properties of the vertically propagating acoustic waves (see, however, Roberts 2006). In the inclined field, in the absence of the mode transformation, the amplitudes of the slow modes that reach the upper boundary are very similar. The propagation of these waves is only affected by the strong gradient in the sound speed, producing partial reflection around $Z=0$ $\mathrm{Mm}$. As the wave propagation is field-aligned, they attack the layer of the strong sound speed gradient with a different angle. We are not aware of any theoretical developments of the wave reflection on the sound speed gradient in the presence of the magnetic field. Based on the analogy with rays in geometrical optics (that applies in the case of high-frequency short spatial wavelength waves) we can speculate that the angle of reflection may depend on the angle of incidence to the reflection layer. This would explain different interference pattern formed by the upcoming and reflected slow waves for the field with different inclinations (compare Fig. 4 a to d).

Fig. 4f, $g$ and $\mathrm{h}$ show the fast magneto-acoustic modes generated by the vertical driver. The relative contribution of the fast mode gets larger with the field inclination $\psi$. Unlike the case of the weak magnetic field, the phase and amplitude of this mode remain constant with height. This happens because the propagation speed of this mode is extremely large and its vertical wavelength is much larger than the size of the simulation domain.

\section{Low-frequency waves in the strong field}

Finally, Fig. 5 and Fig. 7 (panels d and h) illustrate the propagation of the low-frequency waves in the strong magnetic field. This case is qualitatively similar to the high-frequency wave propagation in the strong field, except for the presence of the cut-off layer and a much larger vertical wavelength of waves.

The slow magneto-acoustic waves generated by the driver are now reflected due to the cut-off. This happens at different heights, depending on the field inclination. As follows from the phase dependence on height in Fig. $7 \mathrm{~h}$, a standing wave pattern is formed in the simulations with $\psi=0,30$ and $45^{\circ}$. Unlike this, the $60^{\circ}$ inclined field already decreases the cut-off frequency sufficiently enough to allow for the wave propagation. The phase of the slow-mode waves in the $\psi=60^{\circ}$ case continuously increases with height. Note also, that for the same reason, their relative amplitude increase is more significant that in the case of the slow-mode wave propagation in the less inclined magnetic field. The height of the node layer increases with increasing field inclination. This happens because the waves are reflected at progressively larger heights.

The fast magneto-acoustic waves generated by the driver are identical to the case of $T_{0}=360 \mathrm{~s}$. 

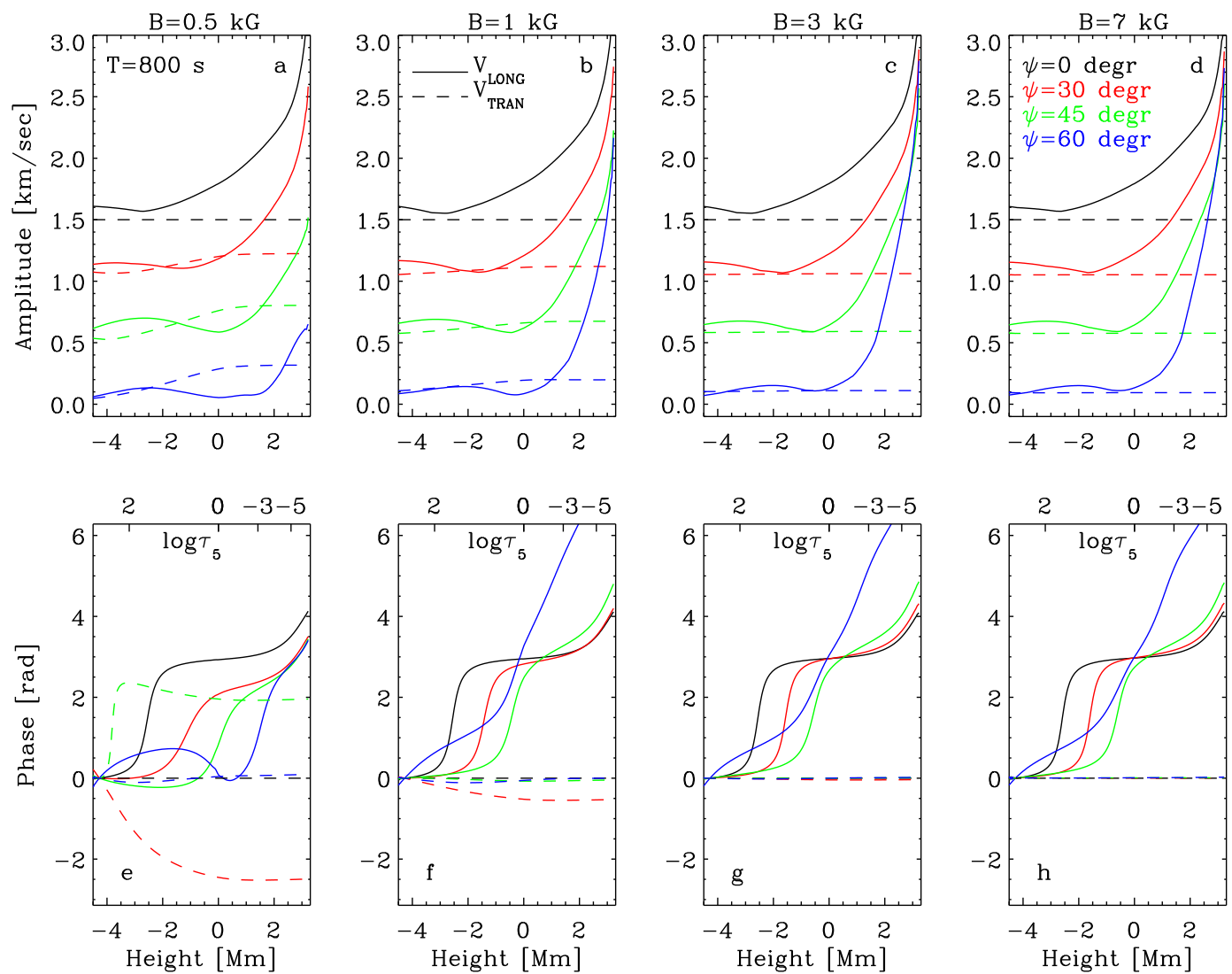

Fig. 7.- Same as Fig. 6 but for $T_{0}=800 \mathrm{~s}$.

\section{Comparative study of the amplitudes and phases}

Figs. 6. 7 and 8 show the height dependence of the velocity amplitudes and phases for the different field strengths and inclinations. The first two figures were partially discussed above. Please recall that, as explained above in Sect. 2.2, the comparison of the amplitudes and phases should be done keeping in mind the particular form of the driver at the lower boundary (i.e. acoustic wave with properties independent on the magnetic field inclination). The relation between the amplitudes of the fast and slow modes for a given inclination $\psi$, as well as the relative amplitudes of waves at different $\psi$ can be modified assuming another form of the driver.

Fig. 6] shows that for the frequencies well above the cut-off frequency $\left(T_{0}=360 \mathrm{~s}\right)$ the field-aligned velocity variations have a character of the upward propagating waves for the field strengths above $1 \mathrm{kG}$. Only for the lowest field strengths in our simulations, $B_{0}=0.5 \mathrm{kG}$, the phase behavior with height is more complex and downward wave propagation is observed for some angles. This complex behavior is produced by the wave transformation and mode mixing at the $c_{S}=v_{A}$ layer. It means that for the weakest fields the high-frequency waves can be trapped, unlike the pure acoustic case. While the vertical propagation is unaltered by the presence of magnetic field, the magnetic effects become more pronounced with increasing inclination. For fields above $1 \mathrm{kG}$ the waves propagate in the atmosphere dominated by the magnetic field and their behavior is more simple. The velocity component perpendicular to the magnetic field shows the behavior characteristic of standing waves for the field strength above $3 \mathrm{kG}$. In general, both amplitude and phase curves get smoother and less node structure is formed with increasing field strength.

Fig. 7 shows that the field-aligned velocity variations for frequencies close to the cut-off frequency $\left(T_{0}=800 \mathrm{~s}\right)$ mostly behave as evanescent trapped waves. The only exception is the situation of the strongly inclined field sufficient to lower down the cut-off frequency. According to Fig. 7, the low-frequency waves are propagating for $\psi=60^{\circ}$. It means that the low-frequency waves that would be trapped in the absence of the magnetic field can escape at latitudes near the magnetic equator if the field strength is sufficiently high (above $B_{0}=0.5 \mathrm{kG}$ in our simulations). Again, due to the mode transformation, the height dependence of the amplitudes and phases is more complex in the $B_{0}=0.5 \mathrm{kG}$ case. The transversal velocity variations show downward wave propagation for the weakest fields and standing waves for the stronger fields.

Velocity variations for the $T_{0}=600 \mathrm{~s}$ period show an intermediate picture between the $T_{0}=800$ and $360 \mathrm{~s}$ cases. In the $B_{0}=0.5 \mathrm{kG}$ field case multiple reflections and phase mixing are present. The cut-off period is just around $600 \mathrm{~s}$ at the very top of the atmosphere (Fig. 1). Because of that the waves in the $\psi=0^{\circ}$ case are reflected and a standing wave pattern is formed. In all other cases the waves are mostly propagating upwards. The amplitude increase with height and the location of the nodes 
is more similar to the $800 \mathrm{~s}$ case, as the wave frequency is closer to the cut-off frequency and the wavelength is much larger than in the $T_{0}=360 \mathrm{~s}$ case.

Fig. 8 give the comparison between the velocity amplitudes at the top of the atmosphere as a function of the field strength for the different inclinations and periods. It provides information of how much of the initial driver energy reaches the top layer and how much of it is retained below due to different processes. First we see that for the vertical field the amplitudes of the longitudinal velocity are field-independent. The absolute values of the amplitudes in the vertical field case decrease with increasing the period. Both properties are in agreement with the analytical models, as the vertically propagating acoustic waves are not affected by the presence of the vertical field. Is gives us an additional confidence in the validity of the simulations.

For the inclined field, the $V_{\text {long }}$ amplitudes depend on the field and this dependence is different for the different periods. For the high-frequency waves $\left(T_{0}=360\right.$ s, left panel of Fig. 8) the amplitudes decrease with the field inclination for all $B_{0}$. This effect is due to nonlinear behaviour of waves. The velocity amplitude of the linear the high-frequency waves scales as $\exp (z / 2 H)$ with height. Preceding the results from Fig. 10, the nonlinearities become important at lower heights for propagation at larger inclination angles. The saw-tooth profile develops and the amplitude increase with height becomes slower than exponential. Thus, in the high-frequency case, the largest amplitudes should be observed near the magnetic poles. This situation is the opposite to the lowfrequency wave case $\left(T_{0}=800 \mathrm{~s}\right.$, right panel of Fig. 8). The velocity amplitude for the linear waves with frequencies below the cut-off frequency scales with height as $\exp \left(z / 2 H-z \sqrt{\left(\omega_{c}^{2}-\omega^{2}\right)} / c_{S}\right)$, making its increase with height weaker than in the high-frequency case. However, as the effective cut-off frequency becomes lower for the waves propagating along the inclined field, the second term in the exponent becomes smaller and finally disappears for sufficiently large inclinations as the waves become propagating again. As follows from the right panel of Fig. 8, this effect is dominating over the effect of non-linearities. Thus, in the low-frequency case the longitudinal velocity amplitudes increase with $\psi$ and are the largest near the equator of the magnetic dipole. The case of $T_{0}=600 \mathrm{~s}$ (middle panel of Fig. 8) is the intermediate between these two. Note that for $\psi=60^{\circ}$, the amplitudes are in fact the same for all $B_{0}$ and $T_{0}$.

For all periods, the amplitudes of the longitudinal velocity at the top of the atmosphere are the lowest for the weakest field strength $B_{0}=0.5 \mathrm{kG}$. At the same time, the amplitudes of the transversal velocity are the highest. This is a direct effect of the mode transformation, as the part of the energy of the fast mode $c_{S}>v_{A}$ waves excited at the lower boundary does not reach the top in the form of slow mode acoustic-like waves but it is transformed to fast mode magnetic waves observed in the transversal component of the velocity. Note that the effects of the mode transformation become more pronounced with increasing inclination, as the fast-to-slow mode transformation becomes less effective. In the $B_{0}=0.5 \mathrm{kG}$ case the dependence of the longitudinal velocity amplitudes on $\psi$ is qualitatively the same for different periods. With increasing field strength, the effects of the mode transformation disappear and the amplitudes become distributed according to the acoustic cut-off frequency effects as described above. It means that, if the field is sufficiently weak and the mode transformation can happen in the atmosphere, the amplitudes of the longitudinal velocity are the largest at the pole of the magnetic dipole, both for the high-frequency and for the low-frequency waves. Note that in all cases the transversal velocity amplitudes at the top of the atmosphere are very small compared to the longitudinal velocity and would contribute very little to the disc-integrated signal.

In summary, in our simulations, all the waves in the weak-field case are partially trapped near the magnetic equator. In the strong-field case, the low-frequency waves are trapped near the poles due to the acoustic cut-off effects, but are propagating near the magnetic equator. The high-frequency waves in the strong field case are not trapped at all magnetic latitudes.

Finally, Figs. 9and 10 describe the non-linear behavior of waves in our simulations. Fig. 9 shows examples of the temporal variations of the longitudinal velocity at the top of the atmosphere $\log \tau_{5}=-5.8$ measured in units of the local sound speed for three different periods. The saw-tooth shape of the temporal profile is evident for the smallest period $T_{0}=360 \mathrm{~s}$. The profile is still asymmetric for $T_{0}=600 \mathrm{~s}$ but is very close to the normal sine-wave shape for $T_{0}=800 \mathrm{~s}$. The amplitudes vary from around $0.25 \times c_{S}$ for $T_{0}=800 \mathrm{~s}$ to $0.7 \times c_{S}$ for $T_{0}=$ $360 \mathrm{~s}$, but stay all the time below the local speed of sound. According to a classical definition of a shock wave, the amplitude of the disturbance should be above the local sound speed. Thus, the saw-tooth waves observed in our simulations are not proper shocks, but simple non-linear disturbances produced after the wave has run a certain distance over the atmosphere.

Fig. 10 gives the height of the saw-tooth wave formation as a function of the magnetic field strength for different inclinations and periods in the simulations. This height was defined in the way explained below. In Fig. 9 we marked three extreme points of the temporal profile $A, B$ and $C$. If the slope between the points $B$ and $C$ is twice steeper than the slope between $A$ and $B$ we say there is a saw-tooth profile. The height of the saw-tooth profile formation depends on the wave period and its amplitude (Priest 1984; Mihalas \& Mihalas 1984) and is typically larger for long-period waves. In our simulations with the initial amplitude of the velocity at the base of the atmosphere of $V_{0}=100 \mathrm{~m} \mathrm{~s}^{-1}$ the saw-tooth profile formation does not occur below $2.2 \mathrm{Mm}$ $\left(\log \tau_{5}=-4.5\right)$. Fig. 10 shows that indeed, the height of the saw-tooth profile formation is the lowest for the lower period waves. The saw-tooth profile formation is less effective for the weakest field as the wave transformation effects prevent the amplitudes from rising sufficiently to produce such profiles. In all the cases (except $B_{0}=0.5$ $\mathrm{kG}$ ), the saw-tooth profile formation height is the lowest at high inclinations $\psi$ because the distance over the which the waves run to reach the same height is larger for the larger inclinations.

\subsection{Variations of thermodynamic parameters and magnetic field}



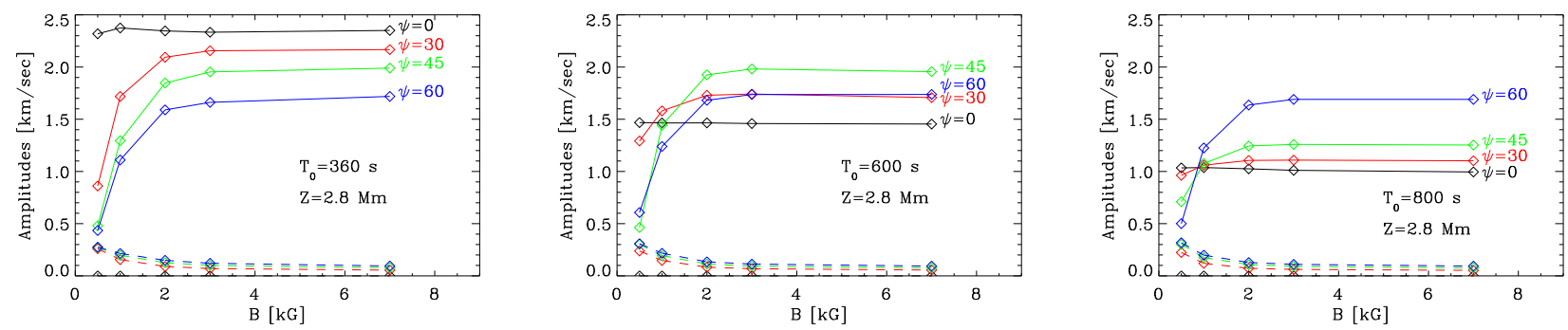

FIG. 8.- Amplitudes measured at the top of the atmosphere at $\mathrm{Z}=2.8 \mathrm{Mm}$ (corresponding to $\log \tau_{5}=-5.2$ ) as a function of the field strength for different inclinations, as indicated on the figure. Left: period $T_{0}=360 \mathrm{~s} ;$ middle: period $T_{0}=600 \mathrm{~s} ;$ right: period $T_{0}=800 \mathrm{~s}$. Solid curves: $V_{\text {long }}$; dashed curves: $V_{\text {tran }}$.

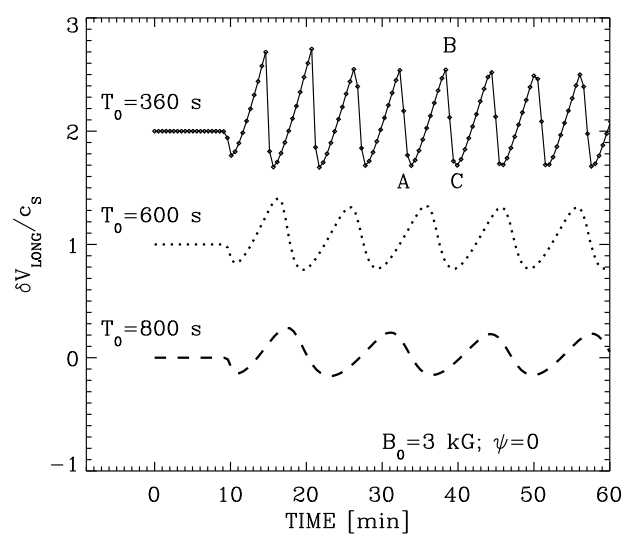

Fig. 9.- Example of the temporal variations of the $V_{\text {long }}$ at $Z=3.2 \mathrm{Mm}\left(\log \tau_{5}=-5.8\right)$ for the simulations with $B_{0}=3 \mathrm{kG}$ $\psi=0$ and three different periods $T_{0}=360,600$ and $800 \mathrm{~s}$. The amplitudes of $V_{\text {long }}$ are measured in units of the local sound speed $c_{S}$. The three curves are separated by $1 \times c_{S}$.

Our simulations allow to calculate self-consistently the variations of the thermodynamic parameters and the magnetic field accompanying the velocity variations. Fig. 11] and Fig. 12 illustrate the pressure, temperature, density and horizontal magnetic field variations for the simulations with $B_{0}=3 \mathrm{kG}$ and $T_{0}=600 \mathrm{~s}$. Unlike the case of the velocity data, we do not show simulations for the extreme cases of $B_{0}$ and $T_{0}$ but rather discuss the intermediate situation. The behavior of the thermodynamic variables and the magnetic field is rather similar in all the simulations with only some quantitative differences.

Fig. 11 shows that the propagation of the pressure, temperature and density disturbances from the deep layers to the surface is not continuous. There is an amplitude and phase jump around $Z=0 \mathrm{Mm}$ height, where the properties of the atmosphere change in an abrupt way. An interesting feature is observed in the density and temperature variations at this layer. There is a very localized amplitude increase (a "bump") present for all field inclinations. The nature of this localized enhancement is probably related to the density jump located around $Z=0 \mathrm{Mm}$. Fig. 12 shows that the amplitudes measured relative to the static values are very high at the "bump" and can reach some 5-10 percent. The phase of perturbations at the "bump" seems to suffer an abrupt $180^{\circ}$ switch. The dependence of the amplitudes at the "bump" on the magnetic field strength $B_{0}$ and inclination $\psi$ is rather complex. For the weak field $\left(B_{0}=0.5\right.$ $\mathrm{kG}$ ) the amplitude of both $\delta T / T$ and $\delta \rho / \rho$ increases with the field inclination. On the other hand, the opposite is true for larger fields $\left(B_{0}>3 \mathrm{kG}\right)$. Note from Fig. 12 that the "bump" is located in the region of formation of continuum intensity around $\log \tau_{5}=1:-1$. Therefore, we can expect that these strong temperature variations will lead to continuum intensity variations which would dominate the total flux changes of the star. The "bump" is absent in the relative pressure variations.

The amplitude of variations of the thermodynamic parameters increases exponentially with height above $\log \tau_{5}=-1$ and reaches some $15-20 \%$ at the top of the atmosphere. In all the cases the amplitudes are smaller for larger inclinations. As follows from the time-height pattern of the pressure and density variations for larger $\psi$ (Fig. 11), at heights above the "bump" both the slow acoustic-like modes and fast magnetic modes contribute to these variations. The standing wave pattern characteristic for the fast modes is especially visible in the $\psi=60^{\circ}$ case at the first $20 \mathrm{~min}$ of the simulations. Interestingly, the presence of the fast mode seems not to affect the temperature variations. Similar to the velocity oscillations, the oscillations of the thermodynamic parameters are more affected by the magnetic field in the weak-field case (Fig. 12, left column of panels). Several node structures are formed in the $\delta P / P$ and $\delta \rho / \rho$ variations for $\psi=45^{\circ}$ and $60^{\circ}$. With increasing field strength, the height variations of the amplitudes become very close for all field inclinations.

The last row of panels in Fig. 11 and Fig. 12 give the time-height plots and the amplitudes of the variations of the horizontal component of the magnetic field vector $\delta B_{x}$. The variations of the magnetic field are not aware of the jump of thermodynamic parameters and their propagation is more continuous. There are no variations of the vertical magnetic field component. The amplitudes of variations of the horizontal magnetic field component are very small and do not exceed 3-4 G. The $\delta B_{x}$ amplitude decreases with increasing the magnetic field strength so that the magnetic field variations are almost absent in the $B_{0}=7 \mathrm{kG}$ case. The $\delta B_{x}$ variations are larger in the deep layers and decrease abruptly above $\log \tau_{5}=-2$. The amplitudes do not have a pronounced dependence on $\psi$. The variations are absent for $\psi=0^{\circ}$ as the wave propagation is purely acoustic-like. 

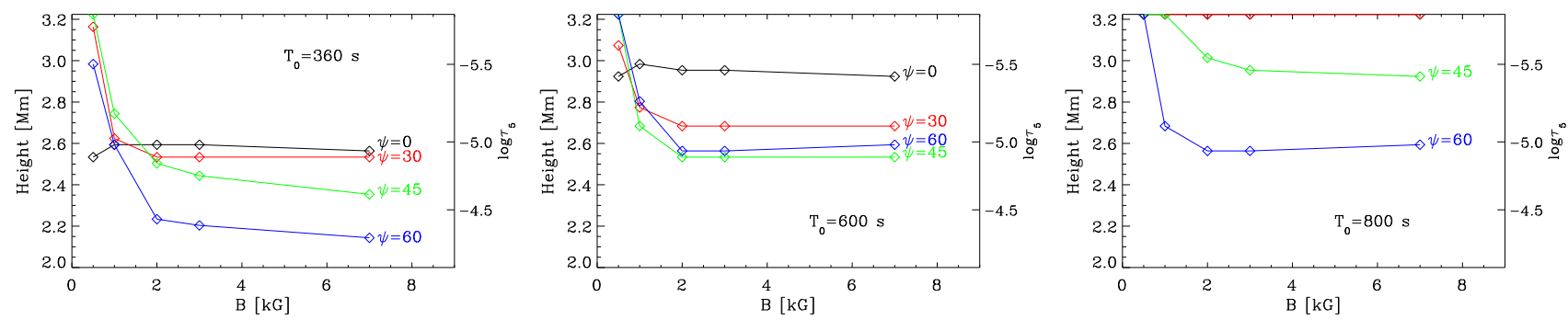

Fig. 10.- Height of the saw-tooth wave formation in $V_{\text {long }}$ as a function of the field strength for different inclinations, as indicated on the figure) . The right vertical axis gives the optical depth $\log \tau_{5}$ for the corresponding geometrical heights. Left: period $T_{0}=360 \mathrm{~s}$; middle: period $T_{0}=600 \mathrm{~s}$; right: period $T_{0}=800 \mathrm{~s}$.
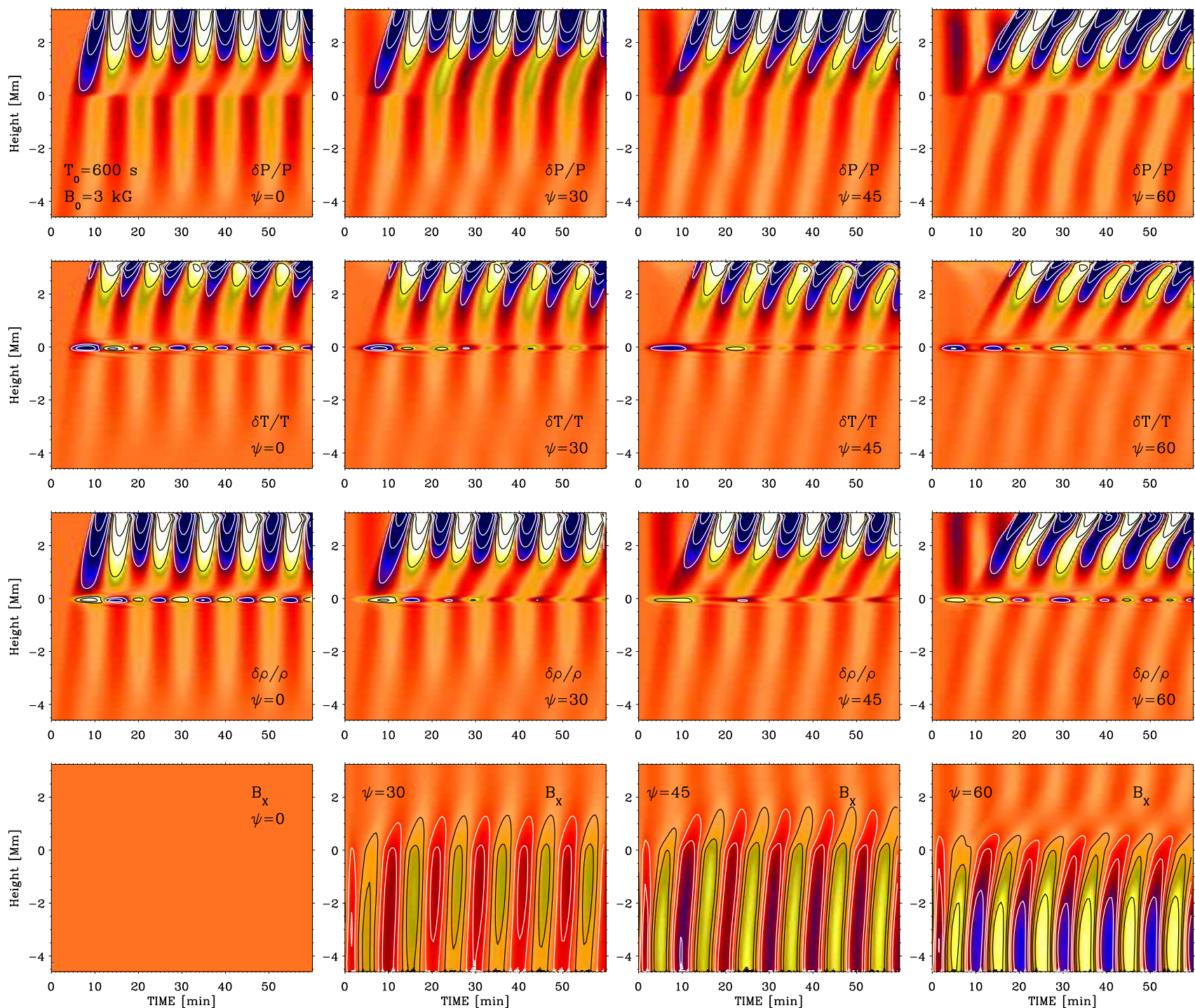

FIG. 11. - Height-time dependences of relative variations of the gas pressure (first row), temperature (second row), density (third row) and horizontal component of the magnetic field vector (last row) for the simulation with $B_{0}=3 \mathrm{kG}$ and $T_{0}=600 \mathrm{~s}$ and inclinations equal $\psi=0,30,45$ and $60^{\circ}$. Yellow and white colors mean positive values while dark red and blue colors mean negative values. The contours of $|\delta P / P|,|\delta T / T|,|\delta \rho / \rho|=(4,10,20) \%$ are plotted as solid lines over the first three panel rows. The contours of $\delta B_{x}=(0.2,0.5,1) \mathrm{G}$ are plotted over the last row of panels. Zero height corresponds to the photospheric base at $\log \tau_{5}=0$.

Finally, Fig. 13 illustrates variations of the gas density, temperature and vertical velocity component at several different optical depths for the representative simulation set $\left(T_{0}=600 \mathrm{~s}, B_{0}=3 \mathrm{kG}, \psi=0^{\circ}\right)$. Unlike the previous height-time and amplitude-height plots, this figure shows absolute variations of the thermodynamic quantities. This format emphasizes strong, anti-phase changes of the temperature and density at the base of 

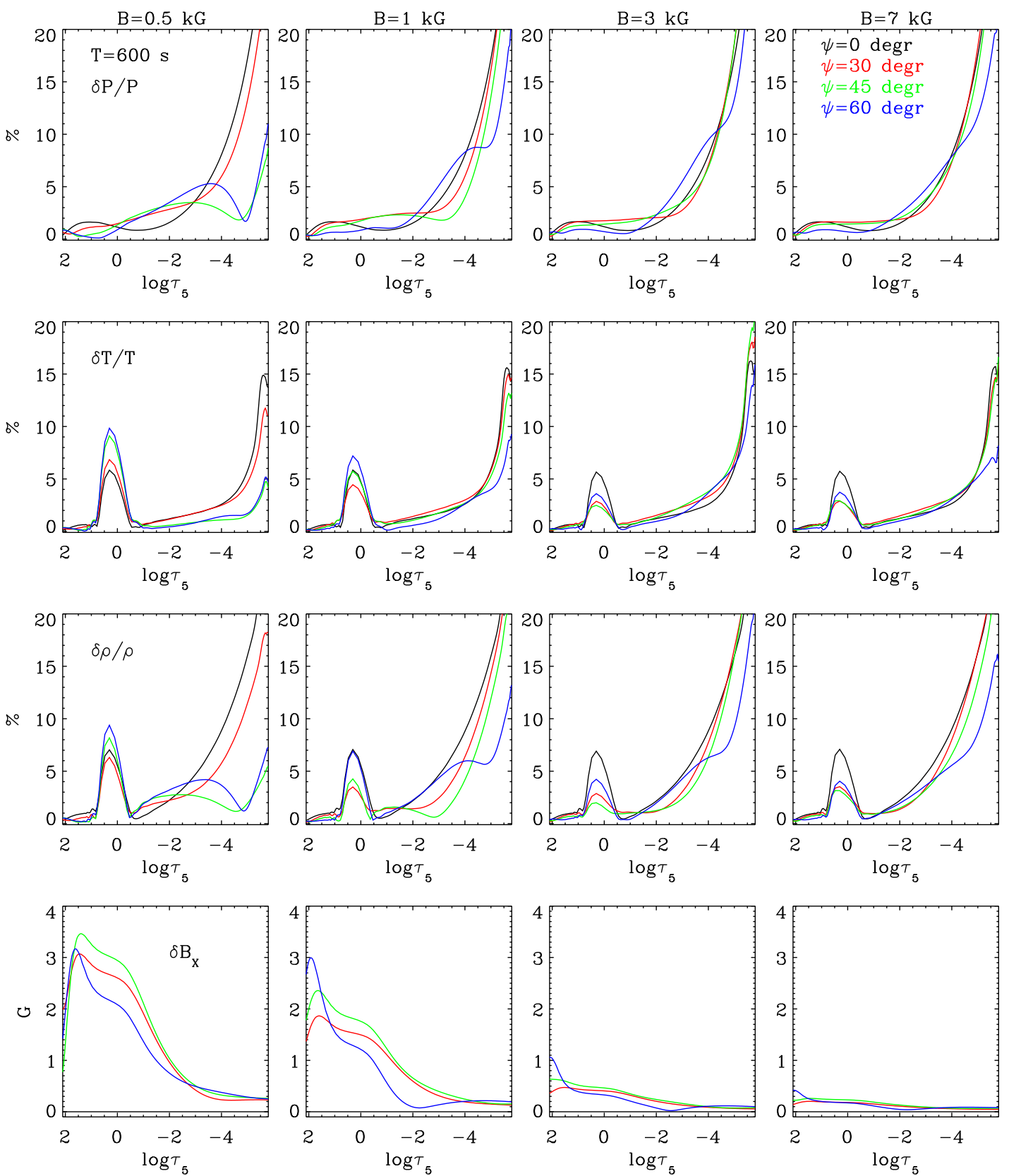

FIG. 12.- Amplitudes of the relative variations of the gas pressure (first row); temperature (second row); density (third row) and horizontal component of the magnetic field vector (last row) as a function of the optical depth $\log \tau_{5}$. Columns from left to right are for the magnetic field strengths $B_{0}=0.5,1,3$ and $7 \mathrm{kG}$. Different curves are for different inclinations, the color coding is indicated in the figure.

the photosphere. In the higher layers the amplitude of the temperature variations first rapidly decreases and then gradually increases again, reaching values comparable to the amplitude at $\log \tau_{5}=0$ only in the uppermost layers. The variation of the vertical velocity component rapidly grows above $\log \tau_{5} \approx-1$ and acquires a noticeable non-sinusoidal shape at $\log \tau_{5}=-5$. In these highest layers the density variation appears to be relatively unimportant. In the upper atmosphere, above $\log \tau_{5}=-1$ the phase shifts between temperature, pressure and density variations follow the behavior characteristic for acoustic-gravity waves dominated by buoyancy force (Mihalas \& Mihalas 1984). The temperature leads downward velocity by some $0.12-0.25$ of pulsation pe- riod $\left(45^{\circ}-90^{\circ}\right)$, depending on height, while the density lags behind the velocity by nearly the same amount. At layers around the "bump" at $\log \tau_{5}=0$, the phase relations are switched by $180^{\circ}$. Of course, details of this pulsation picture depend somewhat on the assumed pulsation period and magnetic field characteristics but the main features persist through all simulations.

\section{GLOBAL MODEL}

In addition to the extensive grid of local simulations for different values of the pulsation period, magnetic field strength and orientation, we performed a more restrictive calculation aimed at reproducing the disc-integrated signal of the oblique non-radial pulsations observed from 


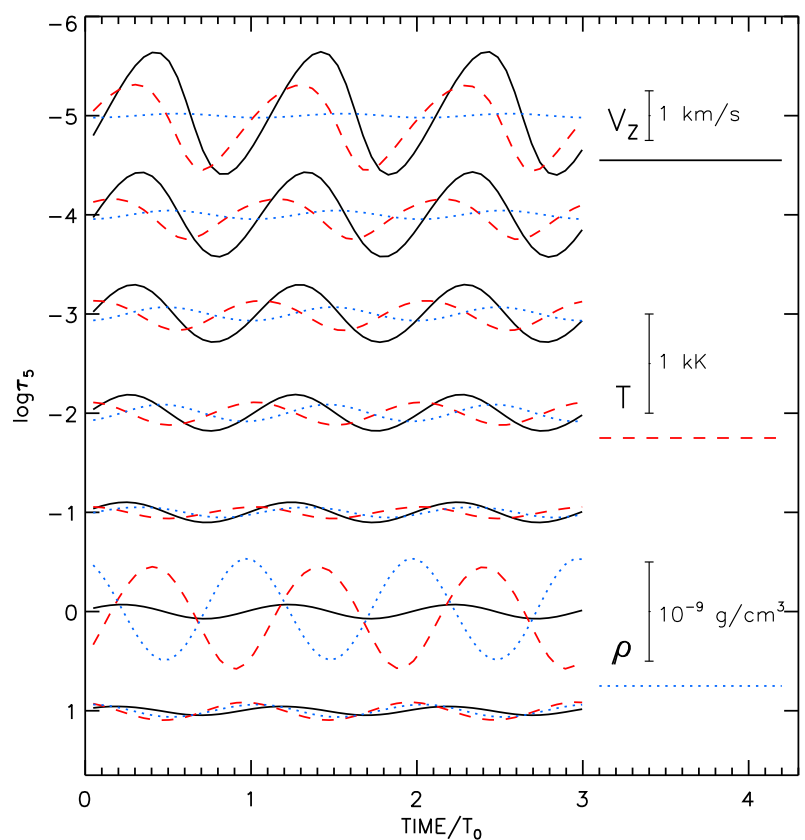

FIG. 13.- Absolute variations of the vertical velocity, temperature and gas density for the simulations with $T_{0}=600 \mathrm{~s}$ and a vertical field with $B_{0}=3 \mathrm{kG}$. Pulsation curves for different optical depths are shifted vertically. Positive velocity direction corresponds to redshift (downflow).

different aspect angles. As discussed in Sect. 1.1, the horizontal structure of the roAp pulsation generally resembles the axisymmetric $\ell=1$ pulsation mode, aligned with the dipolar magnetic field. Therefore, we adopted the field components:

$$
\begin{aligned}
& B_{r}=B_{\mathrm{d}} \cos \theta \\
& B_{\theta}=0.5 B_{\mathrm{d}} \sin \theta,
\end{aligned}
$$

where $\theta$ is the latitude measured from the magnetic pole and $B_{\mathrm{d}}$ is the polar magnetic field strength. The strength and inclination of the magnetic field at latitude $\theta$ are given by:

$$
\begin{aligned}
B_{0} & =\sqrt{B_{r}^{2}+B_{\theta}^{2}} \\
\psi & =\arccos B_{r} / B_{0} .
\end{aligned}
$$

We considered two dipolar field strength values, $B_{\mathrm{d}}=$ 0.8 and $3 \mathrm{kG}$, and adopted the latitude-dependent driver amplitude, $V_{\mathrm{d}}=V_{0} \cos \theta$, according to the simplest form of the oblique pulsator model. Apart from that we did not introduce any latitudinal dependence in the properties of the driver as given by the equations (6 8). As explained in Sect. 2.2 the results of the disc integration are dependent on this particular form of the driver. This should be kept in mind analyzing the disk-integrated variations presented below. Similar to the local models, we used $V_{0}=100 \mathrm{~m} \mathrm{~s}^{-1}$. The driving period was set to $600 \mathrm{~s}$. Simulations were performed for an equidistant latitude grid in $\cos \theta$, varying from $\cos \theta=0.95$ to 0.05 . For the purpose of disk integration, we divided each hemisphere of the star into 10 latitude zones, corresponding to this $\theta$-grid. Each latitude was further partitioned into 60-100 elements, giving us a total of 1148 surface zones.

Four angles are required to fully describe the orientation of the stellar rotation and magnetic axes with respect to the observer (e.g., see Fig. 6 in Piskunov \& Kochukhov 2002). However, here we are not interested in the transverse magnetic field component. Moreover, we limit ourselves to the investigation of the disk average of the line of sight velocity component (radial velocity), which is not altered by the stationary velocity field produced by the stellar rotation Kochukhov 2005). With these simplifications the only relevant parameter is the angle $\gamma$ between the pulsation axis and the line of sight.

We performed the disk integration by transforming the surface pulsation structure from the stellar to the observer's reference frame, rotated the coordinate system according to the angle $\gamma$ and summed, with an appropriate weight, different pulsation quantities over all visible surface elements. This procedure was applied independently for each depth layer and simulation time step. The weight function took into account the projected areas of the surface zones and a linear limb darkening with the coefficient $u=0.5$. We have verified that the line of sight magnetic field component, $B_{\text {los }}$, obtained with this numerical scheme reproduces the longitudinal field calculated with the well-known analytical formula (Leroy et al. 1994) to within 3\%.

Fig. 14 presents the height-time variations of different quantities for the global model with $B_{\mathrm{d}}=3 \mathrm{kG}$. In this plot a positive radial velocity $V_{\text {los }}$ corresponds to the redshift as seen by the observer. On the other hand, a positive longitudinal field $B_{\text {los }}$ points towards the observer, according to the standard definition used in solar and stellar magnetometry.

As expected, the amplitude of pulsations depends on $\gamma$. However, the overall depth dependence is dominated by the pulsations close to the magnetic pole and hence is similar for different angles between the line of sight and the pulsation axis. The radial velocity variations are qualitatively similar to the behavior of $V_{\text {long }}$ for the low $\psi$, strong-field, low-frequency local models (Fig. 51). Thus, the radial velocity pulsations are dominated by the slow acoustic-like modes in all the visible atmospheric layers. This result is easy to understand giving the fact that the amplitudes of the transversal velocity variations produced by the fast magneto-acoustic modes in our simulations are always smaller than the amplitudes of the longitudinal velocity variations due to the slow magnetoacoustic modes, except for the magnetic latitudes with a very inclined magnetic field $\psi=40^{\circ}-60^{\circ}$ (see e.g. Fig. 7). The node structure present in the radial velocity oscillations at $\log \tau_{5}=0$ is the remnant of the nodes due to the wave reflection on the density jump at the photospheric base. This node is persistent at all latitudes and does not disappear in the integrated velocity data. Apart from this node, the wave amplitude in the high layers increases rapidly with height reaching around $1 \mathrm{~km} \mathrm{~s}^{-1}$ at the top of our atmosphere.

Fig. 14 shows that the prominent temperature fluctuations around $\log \tau_{5}=0$ are retained in the diskintegrated picture. Thus, this pulsational feature will have an important influence on the disk-averaged observables. The amplitudes of the temperature fluctuations reach a few percent, a value similar to individual variations at each particular magnetic latitude. The temperature variations are the highest when the pulsation axis and the observational line of sight are co-aligned. 

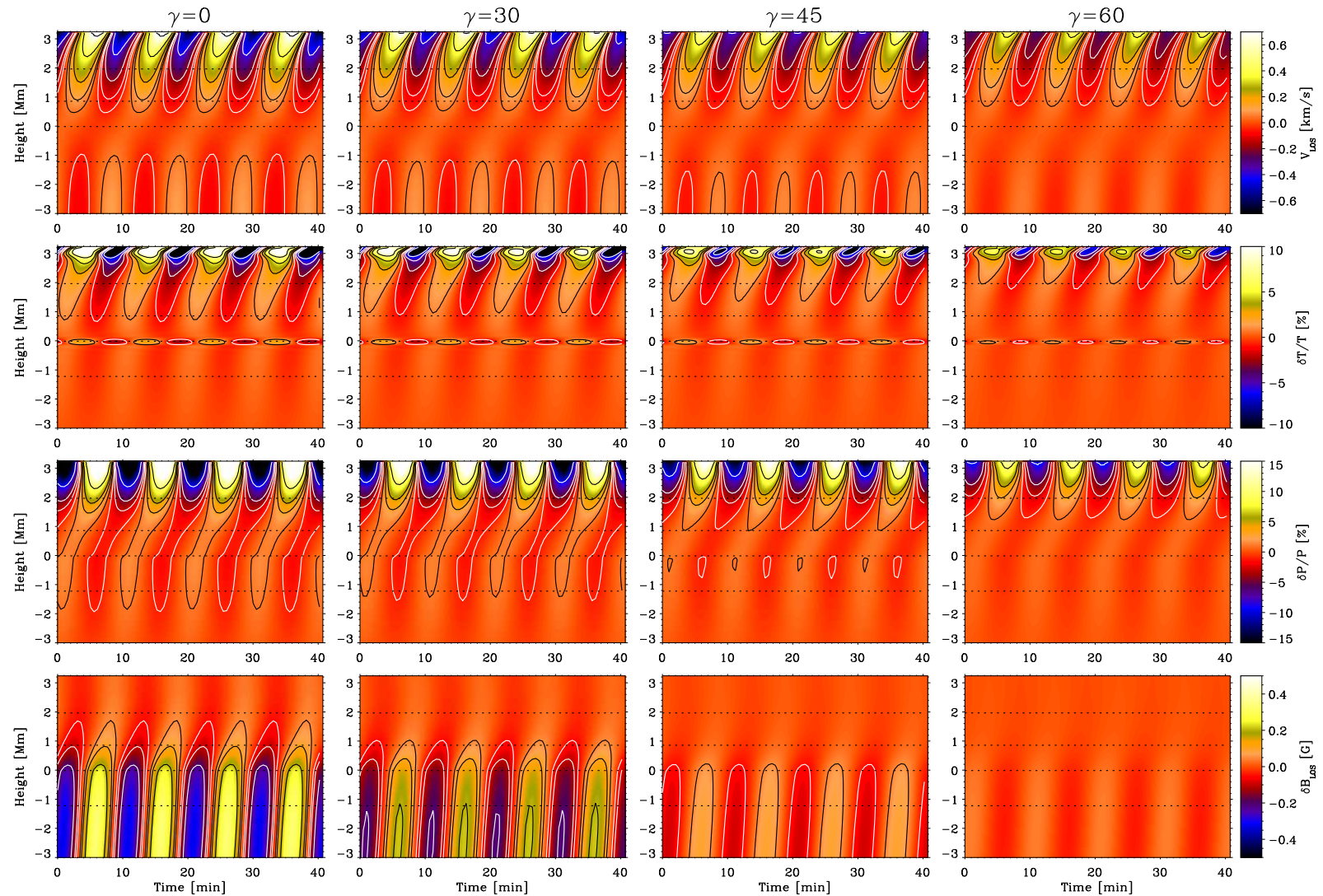

FIG. 14.- Height-time variations of the radial velocity (first row), relative temperature (second row), relative pressure (third row) and the line of sight magnetic field component (bottom row) for the global model with $B_{\mathrm{d}}=3 \mathrm{kG}$ and $T_{0}=600 \mathrm{~s}$. Columns show results for different values of the angle $\gamma$ between the pulsation axis and the line of sight. The contours of $\left|V_{\mathrm{los}}\right|=(0.05,0.1,0.2,0.4,0.8) \mathrm{km} \mathrm{s}{ }^{-1}$, $|\delta T / T|=|\delta P / P|=(1,3,5,8) \%,\left|B_{\text {los }}\right|=(0.05,0.1,0.2,0.4) \mathrm{G}$ are plotted as solid lines. The horizontal dotted lines correspond to the optical depths $\log \tau_{5}=1.5,0,-2$ and -4 .

The variations of the mean field modulus (not shown) exhibit a height dependence comparable to $B_{\text {los }}$ and an amplitude below $1 \mathrm{G}$.

The global model results obtained for the dipolar field with $B_{\mathrm{d}}=0.8 \mathrm{kG}$ are similar to those described above. The main difference is a higher amplitude of the relative temperature variations at the photospheric base (up to $5 \%$ ), an increase of the magnetic field fluctuations by a factor of two and a steeper change of the pulsation velocity phase with depth, reminiscent of the $V_{\text {long }}$ behavior in Fig. 2 ,

\section{DISCUSSION AND CONCLUSIONS}

In this paper we have described the results of the simulations of magneto-acoustic wave propagation in the atmospheres of roAp stars for a wide grid of magnetic field strengths and wave periods. Our simulations allowed us to obtain a more generalized picture of the wave behavior, reflection, mode transformation, formation of evanescent waves and node layers. Our main findings can be summarized in a following way:

- The atmospheric regions above $\log \tau_{5}=0$ of the considered roAp star model are magnetically dominated already for the field strengths as low as 0.5 $\mathrm{kG}$. Both fast and slow magneto-acoustic modes are present simultaneously in these layers. Excited by vertical driving in our simulations, the slow mode waves dominate in the regions of nearly vertical field close to the magnetic poles. The fast mode waves only have comparable amplitudes to the slow mode waves at latitudes close to the magnetic equator, corresponding to $\psi$ larger that $50^{\circ}$ $60^{\circ}$ (depending on the frequency).

- The slow mode waves are mostly propagating upwards and are field-aligned and the fast mode waves acquire a standing wave pattern in the upper atmosphere due to their very large vertical wavelength produced by the rapid increase of the Alfvén speed. This smooth behavior is disturbed by different reflection layers: cut-off layer, $c_{S}=v_{A}$ transformation layer and the density "bump" around $\log \tau_{5}=0$. Due to reflections on (or above) these layers, trapped waves or waves propagating down are produced in the regions below the visible surface in our simulations.

- The node layers can form due to the wave reflections at the cut-off layer, at the density "bump" or after the fast mode reflection above the $c_{S}=v_{A}$ layer. In addition to these physical reasons, another nodes can be formed in the line-of-sight velocity projection due to the interference of the fast and slow magneto-acoustic modes. These type of nodes are more frequent at latitudes close to magnetic equator where the amplitudes of the modes 
become of the same order of magnitude.

- In the atmosphere above $\log \tau_{5}=0$ the amplitude of the slow mode waves increases exponentially with height, in accordance with behavior of acousticgravity waves in a stratified atmosphere. Their phase behavior depends on the frequency and the field inclination. The amplitude and the phase of the fast mode waves vary only slowly with height in agreement with their nearly standing character.

- In the strong-field case, when the whole simulation domain is in the field-dominated regime $\left(B_{0}>1\right.$ $\mathrm{kG}$ ), the low-frequency waves (below the cut-off) are evanescent in the atmosphere, except for the regions close to the magnetic equator, where the cutoff frequency is lowered due to their field-aligned propagation. The high-frequency waves (above the cut-off) are propagating at all magnetic latitudes in the strong-field case. The presence of the mode transformation changes this situation. In the weakfield case $\left(B_{0}<0.5 \mathrm{kG}\right)$ both the high-frequency and low-frequency waves become partially trapped near the magnetic equator due to the reflections of the fast magneto-acoustic mode produced in the magnetically-dominated part of the atmosphere.

- The temperature and density variations show an amplitude and phase jump around $\log \tau_{5}=0$, where the properties of the atmosphere change in an abrupt way. The amplitudes of temperature and density variations are unusually high at these layers and can reach some $5-10 \%$ of the static values. The amplitudes of the magnetic field variations reach at most 3-4 G, decreasing rapidly with height and with the $B_{0}$ field strength of the simulation.

- The disc-integrated variations of the line-of-sight velocity and thermodynamic parameters mostly preserve signatures of the slow magneto-acoustic waves close to the magnetic poles for all angles between the line-of-sight and and pulsation axis. The amplitudes of the variations increase exponentially with height. The node layer in velocity is maintained around $\log \tau_{5}=0$. The prominent increase in the amplitudes of variations of temperature and density around $\log \tau_{5}=0$ is also preserved in the disc-integrated data.

Our conclusions regarding the existence of the fast and slow magneto-acoustic waves and their propagation properties are similar to those obtained in the theoretical work of Sousa \& Cunha (2008). The aim of our analysis was to understand what mechanisms produce such diverse signatures of pulsations measured in the atmospheres of roAp stars. Sousa \& Cunha (2008) addressed a different question, namely the energy losses of the highfrequency waves associated with the presence of magnetic field. Despite the different objectives, our modelling allows us to make some conclusions regarding the behavior of the high-frequency waves as well. In particular, we conclude that, if on their way to the surfaces the high-frequency fast magneto-acoustic waves (essentially acoustic in the interior where $c_{S}>v_{A}$ ) travel through the region of equal Alfvén and sound speeds where the

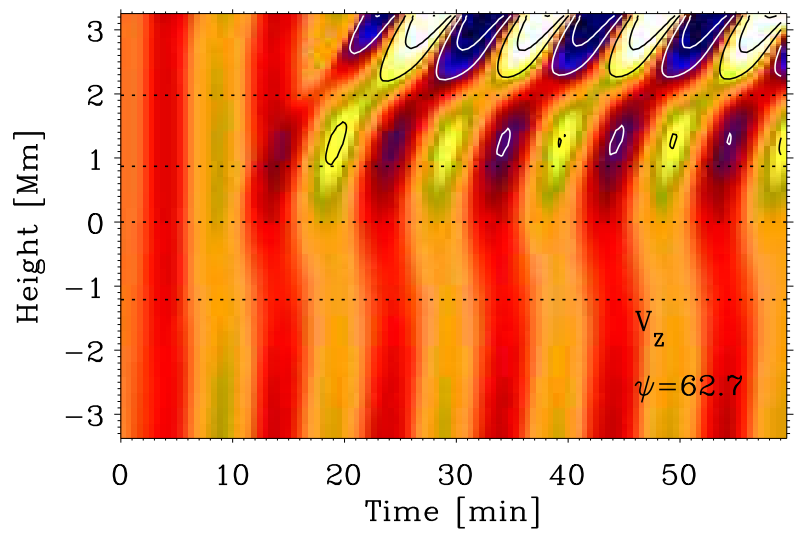

FIG. 15.- Example of the height-time variations of the vertical velocity for the global model with $B_{\mathrm{d}}=3 \mathrm{kG}$ and $T_{0}=600 \mathrm{~s}$ at the magnetic latitude corresponding to $\psi=62.7^{\circ}$. Yellow and white colors mean positive velocity, while dark red and blue colors mean negative velocity. The horizontal dotted lines correspond to the optical depths $\log \tau_{5}=1.5,0,-2$ and -4 . Note the presence of a node around $\log \tau_{5}=-2$ due to the fast and slow mode interference.

mode transformation can occur, they can be partially transmitted as fast magnetic waves in the magnetically dominated atmosphere $\left(c_{S}<v_{A}\right)$. The fast magnetic waves are then reflected back to the interior and thus, some part of their energy is lost at the surface. This way the high-frequency waves can become partially trapped. This mechanism acts more efficiently at high magnetic latitudes. The part of the energy of the acoustic-like fast modes in the interior transmitted as fast magnetic modes increases with increasing the inclination angle between the magnetic field and the direction of the wave propagation, thus becoming larger at the magnetic equator. At the poles where the field is close to vertical, the highfrequency fast acoustic-like modes from the interior are transformed completely into the slow acoustic-like modes in the atmosphere and no trapping occurs.

The difference between our modelling and the one by Sousa \& Cunha (2008) is that we considered much smaller portion of the stellar interior. Thus, in our simulations the wave transformation happens only for the weakest field strengths. Due to that, we have concluded that the high-frequency waves are trapped near the magnetic equator only in the weakest fields, below $0.5 \mathrm{kG}$. If we would have considered a larger portion of the interior of the star, the mode transformation could have affected waves for larger magnetic field strengths as well.

The low-frequency waves with frequencies below the cut-off frequency are shown to be trapped at the magnetic poles due to the cut-off effects. The low-frequency waves are also partially trapped near the magnetic equator, since they are affected by the mode transformation in a similar way as high-frequency waves. However, it is possible that some part of their energy escapes at intermediate magnetic latitudes where the field inclination is already sufficient to lower down the cut-off frequency and the fast to fast mode transmission is not complete. These conclusions are all in line with Sousa \& Cunha (2008).

Our analysis allows us to link directly the theoretically calculated wave perturbations in all quantities to the heights in the atmosphere, and, thus, to compare the 
simulated and observed wave behavior. We stress that the conclusions coming from our analysis remain valid, despite we take only a small portion of the stellar interior. The relative distribution of the wave amplitudes at different frequencies may change due to the mode transformation, but the general picture of the propagation, amplitude increase and the node formation will remain valid. For example, the consequences of the wave reflection (such as the standing wave formation or the downward wave propagation due to the mode transformation) can hardly be detected in observations since these processes happen below the visible surface in the our roAp star model. Only for the weakest field strengths $\left(B_{0}<0.5\right.$ $\mathrm{kG}$ ) these features can, in principle, be detected in the deepest observable atmospheric layers.

Our disc-integrated data suggests that, in the assumed excitation model, the variations of all quantities in the upper atmosphere (except for the magnetic field) are dominated by slow magneto-acoustic modes. This conclusion is different from Sousa \& Cunha (2008) who considered disc-integrated velocity from their simplified, analytical model for the case of the observer being poleon in relation to the magnetic field axis. They suggest that the observed variations are the superposition of the acoustic running waves and magnetic standing waves and the phase depends on the relative contribution of both components. But for the magnetic and pulsation geometry adopted in our study mostly the signatures of the slow acoustic-like waves remain in the disc-integrated data.

One of the main goals of our work is to interpret the complex observational picture of the spectroscopic variability of roAp stars. However, detailed analysis of observations requires computation of the hydrodynamical models and spectrum synthesis for specific stars, taking into account their pulsation frequencies, magnetic field strengths, orientation of pulsation and rotation axes, and individual atmospheric structure. We plan to undertake such an in-depth investigation of selected roAp stars in the future studies. Here we limit ourselves to the qualitative comparison of our model predictions with the main observational features of roAp pulsations.

The growth of the pulsational amplitude with height, observed in all roAp stars, is frequently considered in the context of a simple picture where the kinetic wave energy, $\rho v^{2} / 2$, is approximately constant with height, so that pulsation velocity amplitude increases due to the exponential decrease of density in the stratified atmosphere (e.g., Kurtz et al. 2007). Following these arguments, one can estimate an increase of velocity amplitude by a factor of 5-10 from Fe-peak to REE lines. This is not consistent with the observational studies, which often infer the REE to Fe-peak velocity amplitude ratios of 30-100. Our simulations demonstrate that the variations in the layers probed by the lines of light and iron-peak elements are strongly influenced by the density inversion around $\log \tau_{5}=0$. This structure, located at the base of the photosphere of all dwarf stars in the roAp temperature range, leads to the formation of the velocity node right in the region where lines with weak or undetectable pulsational variation are formed. This node is also present in the disc-integrated data in the same layers, while higher in the atmosphere the amplitude of the line-of-sight velocity increases exponentially. Consequently, as the wave propagates outside the node region, the velocity ampli- tude increases by a factor of 30-50 in our simulations, in reasonable agreement with observations.

The atmospheric structure around $\log \tau_{5}=0$ has equally important repercussions for the pulsational temperature variations. Our simulations predict prominent temperature changes in this region, which will very likely dominate the observed luminosity variation of roAp stars. Thus, our magneto-hydrodynamical models offer a new perspective on the photometric variability of pulsating Ap stars. We suggest that its origin is not a smooth, weak temperature fluctuation, gradually changing with height almost equivalently to $T_{\text {eff }}$ variation (Medupe \& Kurtz 1998), but a high-amplitude, vertically-localized phenomenon. A complete understanding of this temperature variation is crucial for relating spectroscopic and photometric time-resolved observations of roAp stars (Ryabchikova et al. 2007c) and for interpreting photometric pulsations in different bands (Matthews et al. 1996). The position and shape of the density inversion can be also an important factor determining the visibility of the roAp pulsations in the Hertzsprung-Russell diagram. Excitation theories (e.g., Théado et al. 2009) predict roAp-type oscillations in stars much hotter than the empirical blue border of the roAp instability strip at $T_{\text {eff }} \approx 8100 \mathrm{~K}$. It is possible that the absence of the density inversion at the right atmospheric height diminishes luminosity variations, making them undetectable for the photometric pulsation surveys of hotter Ap stars.

The depth dependence of the phase of the velocity variations obtained in our simulations shows a broad agreement with observations. The pulsation phase increases with height, showing the pattern of the outwardrunning wave clearly seen in many roAp stars. The high-frequency models predict a substantial difference in the phase of REE lines formed at different layers in the upper atmosphere. The low-frequency models often demonstrate a slow height variation of the pulsation phase, followed by a more rapid changes in the higher layers. This is reminiscent of the standing-like followed by the running-like wave behaviour reported in observational studies.

For a few roAp stars the radial velocity studies of different REEs reveal a sudden drop of the pulsation amplitude and a $\pi$ radian change in phase at high atmospheric layers. Occasionally, such high-lying node surfaces in the vertical velocity appear in our simulations at high magnetic latitudes. These nodes are produced due to a superposition of the fast and slow magneto-acoustic modes that attain similar amplitudes sufficiently far from the pulsation poles. An example of the vertical velocity variation at the magnetic latitude corresponding to $\psi=62.7^{\circ}$ in the simulations with $B_{\mathrm{d}}=3 \mathrm{kG}$ showing a high-lying node is given in Fig. 15. Though such kind of nodes are quite persistent at high latitudes, they are not retained in the integrated velocity data. It happens because the amplitude of pulsations is small far from the poles for the assumed mode geometry. However, we can speculated that the upper atmospheric nodes can become visible in the radial velocity data due to an inhomogeneous distribution of chemical elements, located in spots close to the magnetic equator. Additionally, contribution of these regions to the disk-integrated signal could be amplified by the magnetic distortion of the mode ge- 
ometry (Saio 2005).

The non-detection of the pulsational variations of magnetic field is in line with the results of our numerical simulations. The disk-integrated amplitudes of the fluctuations of the field modulus and longitudinal field are well below the current detection limits attained in the timeresolved spectroscopic and spectropolarimetric observations of roAp stars. Furthermore, our models show that the depth-dependence of the magnetic field variations is opposite to that of the radial velocity. Minute fluctuations of magnetic field rapidly decrease above $\log \tau_{5}=0$. Thus, an eventual detection of the pulsational variation of magnetic field is far more likely using the lines formed in deep layers, characterized by very weak or undetectable velocity oscillations.

Finally, theoretical pulsation models computed in our study allow us to comment on the REE line profile variation (LPV) of the REE lines forming in the upper atmospheres of roAp stars. This interesting topic was explored in several recent theoretical and observational studies. With the aim to explain the anomalous asymmetric LPV of strong REE lines, Shibahashi et al. (2008) proposed a shock-wave model according to which the intrinsic pulsation velocity amplitude at the REE line formation heights $\left(\log \tau_{5}=-4:-5\right)$ reaches values comparable to the sound speed. To retain an agreement with the observed substantially subsonic, sinusoidal radial velocity curves, Shibahashi et al. postulated that the REE line formation zone spans over a shock-wave train, so that the observed signal corresponds to the vertically-averaged pulsational variation. Our hydrodynamical models do not confirm this picture. Apart from a moderate non-linearity in the uppermost layers, the velocity variations are harmonic and their amplitudes are well below the sound speed. For a broad range of the magnetic inclinations $\left(\psi=0-80^{\circ}\right)$ the REE line-forming zone above $\log \tau_{5}=-2$ accommodates no more than one vertical pulsation wavelength. For very high field inclinations $\left(\psi>80^{\circ}\right)$ multiple nodes can appear in the vertical velocity but the contribution of the corresponding surface regions to the disk-integrated signal is negligible.

An alternative phenomenological model of the pulsational LPV of REE lines (Kochukhov et al. 2007) provides a closer match to the behaviour of our theoretical simulations. Kochukhov et al. showed that the observed LPV can be reproduced by a combination of the subsonic velocity changes and the line width variation. The latter can be related to the temperature fluctuation in the uppermost layers (Fig. 13). However, detailed spectrum synthesis is required to study in detail the LPV predicted by our models and to compare these predictions with the observed pulsational line behaviour.

Detailed spectroscopic analysis and the comparison of the spectra of different chemical elements synthesized in our simulations with observations will be the aim of our future investigations.

EK is grateful to M. Collados for the helpful comments of the manuscript. This research has been funded by the Spanish Ministerio de Educación y Ciencia through projects AYA2007-63881 and AYA2007-66502. OK is a Royal Swedish Academy of Sciences Research Fellow supported by a grant from the Knut and Alice Wallenberg Foundation.

\section{REFERENCES}

Abt, H. A. \& Morrell, N. I. 1995, ApJS, 99, 135

Alecian, G. \& Stift, M. J. 2004, A\&A, 416, 703

Bagnulo, S., Landi degl'Innocenti, E., Landolfi, M., \& Leroy, J. L. 1995, A\&A, 295, 459

Bagnulo, S., Landi Degl'Innocenti, M., Landolfi, M., \& Mathys, G. 2002, A\&A, 394, 1023

Bagnulo, S., Wade, G. A., Donati, J.-F., Landstreet, J. D., Leone, F., Monin, D. N., \& Stift, M. J. 2001, A\&A, 369, 889

Balmforth, N. J., Cunha, M. S., Dolez, N., Gough, D. O., \& Vauclair, S. 2001, MNRAS, 323, 362

Balmforth, N. J. \& Gough, D. O. 1990, ApJ, 362, 256

Berenger, J. P. 1994, J. Comp. Phys., 114, 185

Bigot, L., Provost, J., Berthomieu, G., Dziembowski, W. A., \& Goode, P. R. 2000, A\&A, 356, 218

Bogdan, T. J., Carlsson, M., Hansteen, V., McMurry, A., Rosenthal, C. S., Johnson, M., Petty-Powell, S., Zita, E. J., Stein, R. F., McIntosh, S. W., \& Nordlund, A. 2003, ApJ, 599, 626

Braithwaite, J. \& Nordlund, Å. 2006, A\&A, 450, 1077

Braun, D. C. 1997, ApJ, 487, 447

Cally, P. 2001, ApJ, 548, 473

Cally, P. 2006, Phil. Trans. R. Soc. A, 364, 333

Cally, P. S. \& Bogdan, T. J. 1997, ApJ, 486, L67

Cally, P. S. \& Goossens, M. 2008, Sol. Phys., 251, 251

Cameron, R., Gizon, L., \& Duvall, Jr., T. L. 2008, Sol. Phys., 251, 291

Cunha, M. S. 2006, MNRAS, 365, 153

Cunha, M. S. 2007, Communications in Astroseismology, 150, 48

Cunha, M. S., Fernandes, J. M. M. B., \& Monteiro, M. J. P. F. G. 2003, MNRAS, 343, 831

Cunha, M. S. \& Gough, D. 2000, MNRAS, 319, 1020

Dziembowski, W. A. \& Goode, P. R. 1996, ApJ, 458, 338

Elkin, V. G., Riley, J. D., Cunha, M. S., Kurtz, D. W., \& Mathys, G. 2005, MNRAS, 358, 665

Freyhammer, L. M., Elkin, V. G., Kurtz, D. W., Mathys, G., \& Martinez, P. 2008, MNRAS, 389, 441
Gautschy, A., Saio, H., \& Harzenmoser, H. 1998, MNRAS, 301, 31 Gruberbauer, M., Saio, H., Huber, D., Kallinger, T., Weiss, W. W., Guenther, D. B., Kuschnig, R., Matthews, J. M., Moffat, A. F. J., Rucinski, S., Sasselov, D., \& Walker, G. A. H. 2008, A\&A, 480, 223

Hanasoge, S. M. 2008, ApJ, 680, 1457

Hasan, S. S. \& van Ballegooijen, A. A. 2008, A\&A, 680, 1542

Hasan, S. S., van Ballegooijen, A. A., Kalkofen, W., \& Steiner, O. 2005, ApJ, 631, 1270

Hasan, S. S. \& Ulmschneider, P. 2004, A\&A, 422, 1085

Hubrig, S., Kurtz, D. W., Bagnulo, S., Szeifert, T., Schöller, M., Mathys, G., \& Dziembowski, W. A. 2004a, A\&A, 415, 661

Hubrig, S., Szeifert, T., Schöller, M., Mathys, G., \& Kurtz, D. W. 2004b, A\&A, 415, 685

Kanaan, A. \& Hatzes, A. P. 1998, ApJ, 503, 848

Khomenko, E. 2008, ArXiv e-prints

Khomenko, E., Centeno, R., Collados, M., \& Trujillo Bueno, J. 2008a, ApJ, 676, L85

Khomenko, E. \& Collados, M. 2006, ApJ, 653, 739

Khomenko, E., Collados, M., \& Feliipe, T. 2008b, Solar Phys., 251, 589

Khomenko, E., Kosovichev, A., Collados, M., Parchevsky, K., \& Olshevsky, V. 2009, ApJ, 694, 411

Kochukhov, O. 2004, ApJ, 615, L149

-. 2005, A\&A, 438, 219

-. 2006, A\&A, 446, 1051

Kochukhov, O. 2007, Communications in Astroseismology, 150, 39

Kochukhov, O. 2008, Communications in Asteroseismology, 157, 228

Kochukhov, O. \& Bagnulo, S. 2006, A\&A, 450, 763

Kochukhov, O., Drake, N. A., Piskunov, N., \& de la Reza, R. 2004a, A\&A, 424, 935

Kochukhov, O. \& Ryabchikova, T. 2001, A\&A, 374, 615

Kochukhov, O., Ryabchikova, T., Landstreet, J. D., \& Weiss, W. W. 2004b, MNRAS, 351, L34 
Kochukhov, O., Ryabchikova, T., \& Piskunov, N. 2004c, A\&A, 415, L13

Kochukhov, O., Ryabchikova, T., Weiss, W. W., Landstreet, J. D., \& Lyashko, D. 2007, MNRAS, 376, 651

Kochukhov, O. \& Shulyak, D. 2008, Contributions of the Astronomical Observatory Skalnate Pleso, 38, 419

Kochukhov, O. \& Wade, G. A. 2007, A\&A, 467, 679

Kurtz, D. W. 1982, MNRAS, 200, 807

Kurtz, D. W. 2008, Solar Phys., 251, 21

Kurtz, D. W., Elkin, V. G., Cunha, M. S., Mathys, G., Hubrig, S., Wolff, B., \& Savanov, I. 2006, MNRAS, 372, 286

Kurtz, D. W., Elkin, V. G., \& Mathys, G. 2003, MNRAS, 343, L5 -. 2005, MNRAS, 358, L6

Kurtz, D. W., Elkin, V. G., Mathys, G., \& van Wyk, F. 2007, MNRAS, 381, 1301

Kurtz, D. W. \& Martinez, P. 2000, Baltic Astronomy, 9, 253

Kurtz, D. W., Martinez, P., van Wyk, F., Marang, F., \& Roberts, G. 1994, MNRAS, 268, 641

Landstreet, J. D. \& Mathys, G. 2000, A\&A, 359, 213

Leblanc, F., Monin, D., Hui-Bon-Hoa, A., \& Hauschildt, P. H. 2009, A\&A, 495, 937

Leone, F. \& Kurtz, D. W. 2003, A\&A, 407, L67

Leroy, J. L., Bagnulo, S., Landolfi, M., \& Degl'Innocenti, E. L. 1994, A\&A, 284, 174

Lindsey, C. \& Braun, D. C. 2005a, ApJ, 620, 1107

-. 2005b, ApJ, 620, 1118

Marmolino, C., Severino, G., Deubner, F.-L., \& Fleck, B. 1993, A\&A, 278, 617

Mashonkina, L., Ryabchikova, T., \& Ryabtsev, A. 2005, A\&A, 441, 309

Mashonkina, L., Ryabchikova, T., Ryabtsev, A., \& Kildiyarova, R. 2009, A\&A, 495, 297

Mathys, G., Hubrig, S., Landstreet, J. D., Lanz, T., \& Manfroid, J. 1997, A\&AS, 123, 353

Matthews, J. M., Wehlau, W. H., Rice, J., \& Walker, G. A. H. 1996, ApJ, 459, 278

Medupe, R. \& Kurtz, D. W. 1998, MNRAS, 299, 371

Michaud, G. 1970, ApJ, 160, 641

Michaud, G., Charland, Y., \& Megessier, C. 1981, A\&A, 103, 244

Mihalas, D. \& Mihalas, B. W. 1984, Foundations of Radiation Hydrodynamics (Oxford: Oxford University Press)

Mkrtichian, D. E., Hatzes, A. P., \& Kanaan, A. 2003, MNRAS, 345,781

Moradi, H. \& Cally, P. 2008, Solar Phys., 251, 309

Moradi, H., Hanasoge, S. M., \& Cally, P. S. 2009, ApJ, 690, L72

Moss, D. 2004, in IAU Symposium, Vol. 224, The A-Star Puzzle, ed. J. Zverko, J. Ziznovsky, S. J. Adelman, \& W. W. Weiss, 245-252

Parchevsky, K. V. \& Kosovichev, A. G. 2009, ApJ, 694, 573

Piskunov, N. \& Kochukhov, O. 2002, A\&A, 381, 736

De Pontieu, B., Erdelyi, R. J., \& Stewart, P. 2004, Nat, 430, Issue 6999,536
De Pontieu, B., Hansteen, V. H., Rouppe van der Voort, L., van Noort, M., \& Carlsson, M. 2007, ApJ, 655, 624

Priest, E. R. 1984, Solar magneto-hydrodynamics (Geophysics and Astrophysics Monographs, Dordrecht: Reidel, 1984)

Roberts, B. 2006, Royal Society of London Transactions Series A, 364, Issue 1839, 447

Rosenthal, C. S., Bogdan, T. J., Carlsson, M., Dorch, S. B. F., Hansteen, V., McIntosh, S. W., McMurry, A., Nordlund, A., \& Stein, R. F. 2002, ApJ, 564, 508

Ryabchikova, T., Nesvacil, N., Weiss, W. W., Kochukhov, O., \& Stütz, C. 2004, A\&A, 423, 705

Ryabchikova, T., Piskunov, N., Kochukhov, O., Tsymbal, V., Mittermayer, P., \& Weiss, W. W. 2002, A\&A, 384, 545

Ryabchikova, T., Sachkov, M., Kochukhov, O., \& Lyashko, D. 2007a, A\&A, 473, 907

Ryabchikova, T., Sachkov, M., Weiss, W. W., Kallinger, T., Kochukhov, O., Bagnulo, S., Ilyin, I., Landstreet, J. D., Leone, F., Lo Curto, G., Lüftinger, T., Lyashko, D., \& Magazzù, A. 2007b, A\&A, 462, 1103

-. 2007c, A\&A, 462, 1103

Ryabchikova, T. A., Landstreet, J. D., Gelbmann, M. J., Bolgova, G. T., Tsymbal, V. V., \& Weiss, W. W. 1997, A\&A, 327, 1137

Sachkov, M., Kochukhov, O., Ryabchikova, T., Huber, D., Leone, F., Bagnulo, S., \& Weiss, W. W. 2008, MNRAS, 389, 903

Sachkov, M., Ryabchikova, T., Kochukhov, O., Weiss, W. W., Reegen, P., \& Landstreet, J. D. 2004, in Astronomical Society of the Pacific Conference Series, Vol. 310, IAU Colloq. 193: Variable Stars in the Local Group, ed. D. W. Kurtz \& K. R. Pollard, 208211

Saio, H. 2005, MNRAS, 360, 1022

Saio, H. \& Gautschy, A. 2004, MNRAS, 350, 485

Savanov, I., Hubrig, S., Mathys, G., Ritter, A., \& Kurtz, D. W. 2006, A\&A, 448, 1165

Schunker, H. \& Cally, P. S. 2006, MNRAS, 372, 551

Shibahashi, H., Gough, D., Kurtz, D. W., \& Kambe, E. 2008, PASJ, 60,63

Shibata, K. 1983, PASJ, 35, 263

Shulyak, D., Tsymbal, V., Ryabchikova, T., Stütz, C., \& Weiss, W. W. 2004, A\&A, 428, 993

Sousa, J. C. \& Cunha, M. S. 2008, Journal of Physics Conference Series, 118, 012074

Sousa, S. G. \& Cunha, M. S. 2008, MNRAS, 386, 531

Stępień, K. 2000, A\&A, 353, 227

Steiner, O., Vigeesh, G., Krieger, L., Wedemeyer-Böhm, S., Schaffenberger, W., \& Freytag, B. 2007, Astronomische Nachrichten, 328, 323

Théado, S., Dupret, M.-A., Noels, A., \& Ferguson, J. W. 2009, A\&A, 493, 159

Zhugzhda, Y. D. \& Dzhalilov, N. S. 1982, A\&A, 112, 16

一. 1984, A\&A, 132, 52 